\title{
Hochschild homology relative to a family of groups
}

\author{
ANDREW NICAS \\ DAVID ROSENTHAL
}

\begin{abstract}
We define the Hochschild homology groups of a group ring $\mathbb{Z} G$ relative to a family of subgroups $\mathcal{F}$ of $G$. These groups are the homology groups of a space which can be described as a homotopy colimit, or as a configuration space, or, in the case $\mathcal{F}$ is the family of finite subgroups of $G$, as a space constructed from stratum preserving paths. An explicit calculation is made in the case $G$ is the infinite dihedral group.
\end{abstract}

16E40, 55R35, 19D55

\section{Introduction}

The Hochschild homology of an associative, unital ring $A$ with coefficients in an $A-A$ bimodule $M$ is defined via homological algebra by $H H_{*}(A, M):=\operatorname{Tor}_{*}^{A \otimes A^{\mathrm{op}}}(M, A)$, where $A^{\text {op }}$ is the opposite ring of $A$. In the case $A=\mathbb{Z} G$, the integral group ring of a discrete group $G$, and $M=\mathbb{Z} G$, the Hochschild homology groups $H H_{*}(\mathbb{Z} G):=$ $H H_{*}(\mathbb{Z} G, \mathbb{Z} G)$ have the following homotopy theoretic description. The cyclic bar construction associates to a group $G$ a simplicial set $N^{\text {cyc }}(G)$ whose homology is $H H_{*}(\mathbb{Z} G)$. Viewing $G$ as a category, $\mathbf{G}$, consisting of a single object and with morphisms identified with the elements of $G$, consider the functor $N$ from $\mathbf{G}$ to the category of sets given by $N(*)=G$ and, for a morphism $g \in G=\operatorname{Mor}_{\mathbf{G}}(*, *)$, the map $N(g): G \rightarrow G$ is conjugation, sending $x$ to $g^{-1} x g$. The geometric realization of $N^{\text {cyc }}(G)$ is homotopy equivalent to hocolim $N$, the homotopy colimit of $N$. There is also a natural homotopy equivalence $\left|N^{\mathrm{cyc}}(G)\right| \rightarrow \mathcal{L}(\mathrm{B} G)$ (see Loday [12, Theorem 7.3.11]), where $\mathrm{B} G$ is the classifying space of $G$ and $\mathcal{L}(\mathrm{B} G)$ is the free loop space of $\mathrm{B} G$, ie, the space of continuous maps of the circle into $\mathrm{B} G$. In particular, there are isomorphisms:

$$
H H_{*}(\mathbb{Z} G) \cong H_{*}(\operatorname{hocolim} N) \cong H_{*}(\mathcal{L}(\mathrm{B} G)) .
$$

A family of subgroups of a group $G$ is a nonempty collection of subgroups of $G$ that is closed under conjugation and finite intersections. In this paper we define the Hochschild homology of a group ring $\mathbb{Z} G$ relative to a family of subgroups $\mathcal{F}$ of $G$, denoted $H H_{*}^{\mathcal{F}}(\mathbb{Z} G)$. This is accomplished at the level of spaces. We define a functor 
$N_{\mathcal{F}}: \operatorname{Or}(G, \mathcal{F}) \rightarrow \mathrm{CGH}$ where $\operatorname{Or}(G, \mathcal{F})$ is the orbit category of $G$ with respect to $\mathcal{F}$ and CGH is the category of compactly generated Hausdorff spaces. By definition, $H H_{*}^{\mathcal{F}}(\mathbb{Z} G):=H_{*}\left(\right.$ hocolim $\left.N_{\mathcal{F}}\right)$. If $\mathcal{F}$ is the trivial family, ie, contains only the trivial group, then $N \cong N_{\mathcal{F}}$ and so $H H_{*}^{\mathcal{F}}(\mathbb{Z} G)=H H_{*}(\mathbb{Z} G)$.

For a discrete group $G$ and any family $\mathcal{F}$, let $\mathrm{E}_{\mathcal{F}} G$ be a universal space for $G$-actions with isotropy in $\mathcal{F}$. That is, $\mathrm{E}_{\mathcal{F}} G$ is a $G-\mathrm{CW}$ complex whose isotropy groups belong to $\mathcal{F}$ and for every $H$ in $\mathcal{F}$, the fixed point set $\left(\mathrm{E}_{\mathcal{F}} G\right)^{H}$ is contractible. Given a $G$-space $X$, let $F(X)$ be the configuration space of pairs of points in $X$ which lie on the same $G$-orbit. This space inherits a $G$-action via restriction of the diagonal action of $G$ on $X \times X$.

Suppose that $G$ is countable and that the family $\mathcal{F}$ of subgroups is also countable.

Theorem A There is a natural homotopy equivalence hocolim $N_{\mathcal{F}} \simeq G \backslash F\left(\mathrm{E}_{\mathcal{F}} G\right)$.

Indeed, this homotopy equivalence is a homeomorphism for an appropriate model of the homotopy colimit (see Theorem 3.7 and Corollary 3.8).

Specializing to the case where $\mathcal{F}$ is the family of finite subgroups of $G$, we write $\underline{\mathrm{E}} G:=\mathrm{E}_{\mathcal{F}} G$ and $\underline{\mathrm{B}} G:=G \backslash \underline{\mathrm{E}} G$. Let $P_{\mathrm{sp}}^{\mathrm{m}}(\underline{\mathrm{B}} G)$ denote the space of marked stratum preserving paths in $\underline{\mathrm{B}} G$ consisting of stratum preserving paths in $\underline{\mathrm{B}} G$ (with the orbit type partition) whose endpoints are "marked" by an orbit of the diagonal action of $G$ on $\underline{\mathrm{E}} G \times \underline{\mathrm{E}} G$. We show (see Theorem 4.26(i)):

Theorem B There is a natural homotopy equivalence hocolim $N_{\mathcal{F}} \simeq P_{\mathrm{sp}}^{\mathrm{m}}(\underline{\mathrm{B}} G)$.

Theorem B is a consequence of Theorem A and a homotopy equivalence $G \backslash F(X) \simeq$ $P_{\mathrm{sp}}^{\mathrm{m}}(G \backslash X)$, which is valid for any proper $G-\mathrm{CW}$ complex $X$ (see Theorem 4.20). The Covering Homotopy Theorem of Palais (Theorem 4.7) plays a key role in the proof of the latter result.

If $\underline{\mathrm{E}} G$ satisfies a certain isovariant homotopy theoretic condition then $P_{\mathrm{sp}}^{\mathrm{m}}(\underline{\mathrm{B}} G)$ is homotopy equivalent to a subspace $\mathcal{L}_{\mathrm{sp}}^{\mathrm{m}}(\underline{\mathrm{B}} G) \subset P_{\mathrm{sp}}^{\mathrm{m}}(\underline{\mathrm{B}} G)$, which we call the marked stratified free loop space of $\underline{\mathrm{B}} G$ (see Theorem 4.26(ii)). We show that this condition is satisfied for appropriate models of $\underline{E} G$ in the following cases:

(1) $G$ is torsion free (see Remark 4.25); note that in this case $\underline{\mathrm{E}} G=\mathrm{E} G$, a universal space for free proper $G$-actions.

(2) $G$ belongs to a particular class of groups that includes the infinite dihedral group and hyperbolic or Euclidean triangle groups (see Example 5.5 and Example 5.6). 
(3) finite products of such groups (see Remark 5.7).

When $G$ is torsion free, $\mathcal{L}_{\mathrm{sp}}^{\mathrm{m}}(\underline{\mathrm{B}} G)$ is homeomorphic to $\mathcal{L}(\mathrm{B} G)$ by Proposition 4.22 and so our result can be viewed as a generalization of the homotopy equivalence $\left|N^{\text {cyc }}(G)\right| \simeq \mathcal{L}(\mathrm{B} G)$

There is an equivariant map $\mathrm{E} G \rightarrow \underline{\mathrm{E}} G$ that is unique up to equivariant homotopy. It induces a map $G \backslash F(\mathrm{E} G) \rightarrow G \backslash F(\underline{\mathrm{E}} G)$, equivalently, a map hocolim $N \rightarrow$ hocolim $N_{\mathcal{F}}$, where $\mathcal{F}$ is the family of finite subgroups of $G$. We explicitly compute this map in the case $G=D_{\infty}$, the infinite dihedral group. In particular, this yields a computation of the homomorphism $H H_{*}\left(\mathbb{Z} D_{\infty}\right) \rightarrow H H_{*}^{\mathcal{F}}\left(\mathbb{Z} D_{\infty}\right)$ (see Section 6).

The paper is organized as follows. In Section 1 we review some aspects of the theory of homotopy colimits. The functor $N_{\mathcal{F}}: \operatorname{Or}(G, \mathcal{F}) \rightarrow \mathrm{CGH}$ is defined in Section 2, thus yielding the space $\mathfrak{N}(G, \mathcal{F}):=$ hocolim $N_{\mathcal{F}}$, which we call the Hochschild complex of $G$ with respect to the family of subgroups $\mathcal{F}$. In Section 3 we study the configuration space $F(X)$ in a general context and give an alternative description of $\mathfrak{N}(G, \mathcal{F})$ as the orbit space $G \backslash F\left(\mathrm{E}_{\mathcal{F}} G\right)$. The homotopy equivalence $G \backslash F(X) \simeq P_{\mathrm{sp}}^{\mathrm{m}}(G \backslash X)$, for any proper $G-\mathrm{CW}$ complex $X$, is established in Section 4 . We also show in this section that if $\underline{\mathrm{E}} G$ satisfies a certain isovariant homotopy theoretic condition, then $P_{\mathrm{sp}}^{\mathrm{m}}(\underline{\mathrm{B}} G)$ is homotopy equivalent to the subspace $\mathcal{L}_{\mathrm{sp}}^{\mathrm{m}}(\underline{\mathrm{B}} G) \subset P_{\mathrm{sp}}^{\mathrm{m}}(\underline{\mathrm{B}} G)$. In Section 5 we show that this condition is satisfied for a class of groups that includes the infinite dihedral group and hyperbolic or Euclidean triangle groups. In Section 6 we analyze the map $G \backslash F(\mathrm{E} G) \rightarrow G \backslash F(\underline{\mathrm{E} G})$, and compute it explicitly in the case $G=D_{\infty}$ thereby obtaining a computation of the homomorphism $H H_{*}\left(\mathbb{Z} D_{\infty}\right) \rightarrow H H_{*}^{\mathcal{F}}\left(\mathbb{Z} D_{\infty}\right)$.

Acknowledgements Andrew Nicas is partially supported by a grant from the Natural Sciences and Engineering Research Council of Canada. The authors thank the referee for useful comments.

\section{Homotopy colimits and spaces over a category}

In this section we provide some categorical preliminaries, following Davis and Lück [7], that will be used in Section 2 to define a Hochschild complex associated to a family of subgroups. Throughout Sections 1 and 2 we work in the category of compactly generated Hausdorff spaces, denoted by $\mathrm{CGH} .{ }^{1}$

\footnotetext{
${ }^{1}$ Given a Hausdorff space $Y$, the associated compactly generated space $\mathrm{k} Y$ is the space with the same underlying set and with the topology defined as follows: a closed set of $\mathrm{k} Y$ is a set that meets each compact set of $Y$ in a closed set. $Y$ is an object of $\mathrm{CGH}$ if and only if $Y=\mathrm{k} Y$, ie, $Y$ is compactly
} 
Let $\mathcal{C}$ be a small category. A covariant (contravariant) $\mathcal{C}$-space, is a covariant (contravariant) functor from $\mathcal{C}$ to $\mathrm{CGH}$. If $X$ is a contravariant $\mathcal{C}$-space and $Y$ is a covariant $\mathcal{C}$-space, then their tensor product is defined by

$$
X \otimes_{\mathcal{C}} Y=\coprod_{C \in \operatorname{obj}(\mathcal{C})} X(C) \times Y(C) / \sim
$$

where $\sim$ is the equivalence relation generated by

$$
(X(\phi)(x), y) \sim(x, Y(\phi)(y))
$$

for all $\phi \in \operatorname{Mor}_{\mathcal{C}}(C, D), x \in X(D)$ and $y \in Y(C)$.

A map of $\mathcal{C}$-spaces is a natural transformation of functors. Given a $\mathcal{C}$-space $X$ and a topological space $Z$, let $X \times Z$ be the $\mathcal{C}$-space defined by $(X \times Z)(C)=X(C) \times Z$, where $C$ is an object in $\mathcal{C}$. Two maps of $\mathcal{C}$-spaces $\alpha, \beta: X \rightarrow X^{\prime}$ are $\mathcal{C}$-homotopic if there is a natural transformation $H: X \times[0,1] \rightarrow X^{\prime}$ such that $\left.H\right|_{X \times\{0\}}=\alpha$ and $\left.H\right|_{X \times\{1\}}=\beta$. A map $\alpha: X \rightarrow X^{\prime}$ is a $\mathcal{C}$-homotopy equivalence if there is a map of $\mathcal{C}$-spaces $\beta: X^{\prime} \rightarrow X$ such that $\alpha \beta$ is $\mathcal{C}$-homotopic to $\mathrm{id}_{X^{\prime}}$ and $\beta \alpha$ is $\mathcal{C}$-homotopic to $\operatorname{id}_{X}$. The map $\alpha: X \rightarrow X^{\prime}$ is a weak $\mathcal{C}$-homotopy equivalence if for every object $C$ in $\mathcal{C}$, the map $\alpha(C): X(C) \rightarrow X^{\prime}(C)$ is an ordinary weak homotopy equivalence. Two $\mathcal{C}$-spaces $X$ and $X^{\prime}$ are $\mathcal{C}$-homeomorphic if there are maps $\alpha: X \rightarrow X^{\prime}$ and $\alpha^{\prime}: X^{\prime} \rightarrow X$ such that $\alpha^{\prime} \alpha=\operatorname{id}_{X}$ and $\alpha \alpha^{\prime}=\operatorname{id}_{X^{\prime}}$. If $X$ and $X^{\prime}$ are $\mathcal{C}$-homeomorphic contravariant $\mathcal{C}$-spaces and $Y$ and $Y^{\prime}$ are $\mathcal{C}$-homeomorphic covariant $\mathcal{C}$-spaces, then $X \otimes_{\mathcal{C}} Y$ is homeomorphic to $X^{\prime} \otimes_{\mathcal{C}} Y^{\prime}$.

A contravariant free $\mathcal{C}-C W$ complex $X$ is a contravariant $\mathcal{C}$-space $X$ together with a filtration

$$
\varnothing=X_{-1} \subset X_{0} \subset X_{1} \subset \cdots \subset X_{n} \subset \cdots \subset X=\bigcup_{n \geq 0} X_{n}
$$

such that $X=\operatorname{colim}_{n \rightarrow \infty} X_{n}$ and for any $n \geq 0$, the $n-$ skeleton, $X_{n}$, is obtained from the $(n-1)$-skeleton, $X_{n-1}$, by attaching free contravariant $\mathcal{C}-n$-cells. That is, there is a pushout of $\mathcal{C}$-spaces of the form

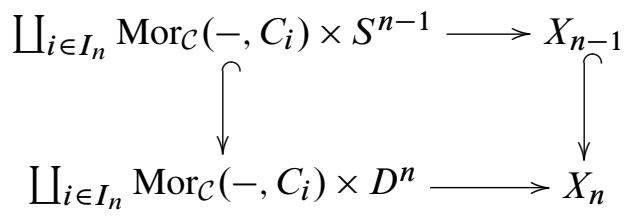

generated. The product of two spaces $Y$ and $Z$ in CGH is defined by $Y \times Z:=\mathrm{k}(Y \times Z)$, where $Y \times Z$ on the right side has the product topology. Function space topologies in $\mathrm{CGH}$ are defined by applying $\mathrm{k}$ to the compact-open topology. In Section 3 and Section 4 we work in the category TOP of all topological spaces and will have occasion to compare the topologies on $Y$ and $\mathrm{k} Y$ (see Proposition 3.6). 
where $I_{n}$ is an indexing set and $C_{i}$ is an object in $\mathcal{C}$. A covariant free $\mathcal{C}-C W$ complex is defined analogously, the only differences being that the $\mathcal{C}$-space is covariant and the $\mathcal{C}$-space $\operatorname{Mor}_{\mathcal{C}}\left(C_{i},-\right)$ is used in the pushout diagram instead of $\operatorname{Mor}_{\mathcal{C}}\left(-, C_{i}\right)$.

A free $\mathcal{C}-\mathrm{CW}$ complex should be thought of as a generalization of a free $G-\mathrm{CW}$ complex. The two notions coincide if $\mathcal{C}$ is the category associated to the group $G$, ie, the category with one object and one morphism for every element of $G$.

Let $\mathrm{EC}$ be a contravariant free $\mathcal{C}-\mathrm{CW}$ complex such that $\mathrm{EC}(C)$ is contractible for every object $C$ of $\mathcal{C}$. Such a $\mathcal{C}$-space always exists and is unique up to homotopy type [7, Section 3]. One particular example is defined as follows.

Let $\mathrm{B}^{\mathrm{bar}} \mathcal{C}$ be the bar construction of the classifying space of $\mathcal{C}, \mathrm{ie}, \mathrm{B}^{\mathrm{bar}} \mathcal{C}=|N \cdot \mathcal{C}|$, the geometric realization of the nerve of $\mathcal{C}$. Let $C$ be an object in $\mathcal{C}$. The undercategory, $C \downarrow \mathcal{C}$, is the category whose objects are pairs $(f, D)$, where $f: C \rightarrow D$ is a morphism in $\mathcal{C}$, and whose morphisms, $p:(f, D) \rightarrow\left(f^{\prime}, D^{\prime}\right)$, consist of a morphism $p: D \rightarrow D^{\prime}$ in $\mathcal{C}$ such that $p \circ f=f^{\prime}$. Notice that a morphism $\phi: C \rightarrow C^{\prime}$ induces a functor $\phi^{*}:\left(C^{\prime} \downarrow \mathcal{C}\right) \rightarrow(C \downarrow \mathcal{C})$ defined by $\phi^{*}(f, D)=(f \circ \phi, D)$. Let $\mathrm{E}^{\text {bar }} \mathcal{C}: \mathcal{C} \rightarrow$ CGH be the contravariant functor defined by:

$$
\begin{aligned}
& \mathrm{E}^{\mathrm{bar}} \mathcal{C}(C)=\mathrm{B}^{\mathrm{bar}}(C \downarrow \mathcal{C}) \\
& \mathrm{E}^{\mathrm{bar}} \mathcal{C}\left(\phi: C \rightarrow C^{\prime}\right)=\mathrm{B}^{\mathrm{bar}}\left(\phi^{*}\right)
\end{aligned}
$$

This is a model for $\mathrm{EC}$. Moreover, $\mathrm{E}^{\text {bar }} \mathcal{C} \otimes_{\mathcal{C}} *$ is homeomorphic to $\mathrm{B}^{\mathrm{bar}} \mathcal{C}$ [7, Section 3].

Lemma 1.1 [7, Lemma 1.9] Let $F: \mathcal{D} \rightarrow \mathcal{C}$ be a covariant functor, $Z$ a covariant $\mathcal{D}$-space and $X$ a contravariant $\mathcal{C}$-space. Let $F_{*} Z$ be the covariant $\mathcal{C}$-space $\operatorname{Mor}_{\mathcal{C}}\left(F\left(-_{\mathcal{D}}\right),-_{\mathcal{C}}\right) \otimes_{\mathcal{D}} Z$, where $-_{\mathcal{C}}$ denotes the variable in $\mathcal{C}$ and $-_{\mathcal{D}}$ denotes the variable in $\mathcal{D}$. Then

$$
X \otimes_{\mathcal{C}} F_{*} Z \rightarrow(X \circ F) \otimes_{\mathcal{D}} Z
$$

is a homeomorphism.

Proof The map $e: X \otimes_{\mathcal{C}}\left(\operatorname{Mor}_{\mathcal{C}}\left(F(-\mathcal{D}),-_{\mathcal{C}}\right) \otimes_{\mathcal{D}} Z\right) \longrightarrow(X \circ F) \otimes_{\mathcal{D}} Z$ is defined by

$$
e([x,[f, y]])=[X(f)(x), y],
$$

where $x \in X(C), y \in Z(D)$ and $f \in \operatorname{Mor}_{\mathcal{C}}(F(D), C)$, for objects $C$ in $\mathcal{C}$ and $D$ in $\mathcal{D}$. The inverse is given by mapping $[w, z] \in(X \circ F) \otimes_{\mathcal{D}} Z$ to $\left[w,\left[\operatorname{id}_{F(D)}, z\right]\right]$, where $w \in(X \circ F)(D)$ and $z \in Z(D)$. 
Definition 1.2 Let $Y$ be a covariant $\mathcal{C}$-space. Then

$$
\underset{\mathcal{C}}{\operatorname{hocolim}} Y:=\mathrm{E}^{\mathrm{bar}} \mathcal{C} \otimes_{\mathcal{C}} Y \text {. }
$$

A map $\alpha: Y \rightarrow Y^{\prime}$ of $\mathcal{C}$-spaces induces a map $\alpha_{*}: \operatorname{hocolim}_{\mathcal{C}} Y \rightarrow \operatorname{hocolim}_{\mathcal{C}} Y^{\prime}$. If $*$ is the $\mathcal{C}$-space that sends every object to a point, then

$$
\underset{\mathcal{C}}{\operatorname{hocolim}} *=\mathrm{E}^{\text {bar }} \mathcal{C} \otimes_{\mathcal{C}} * \cong \mathrm{B}^{\text {bar }} \mathcal{C} .
$$

Therefore, the collapse map, $Y \rightarrow *$, induces a map $\bar{\pi}$ : $\operatorname{hocolim}_{\mathcal{C}} Y \rightarrow \mathrm{B}^{\text {bar }} \mathcal{C}$.

There are several well-known constructions for the homotopy colimit, each yielding the same space up to homotopy equivalence (see Talbert [22, Theorem 1.2]). In particular, using the transport category, $\mathcal{T}_{\mathcal{C}}(Y)$, one can define the homotopy colimit of $Y$ to be $\mathrm{B}^{\text {bar }} \mathcal{T}_{\mathcal{C}}(Y)$. Recall that an object of $\mathcal{T}_{\mathcal{C}}(Y)$ is a pair $(C, x)$, where $C$ is an object of $\mathcal{C}$ and $x \in Y(C)$, and a morphism $\phi:(C, x) \rightarrow\left(C^{\prime}, x^{\prime}\right)$ is a morphism $\phi: C \rightarrow C^{\prime}$ in $\mathcal{C}$ such that $Y(\phi)(x)=x^{\prime}$. The following lemma shows that $\mathrm{B}^{\text {bar }} \mathcal{T}_{\mathcal{C}}(Y)$ is not only homotopy equivalent to our definition of the homotopy colimit of $Y$, but is in fact homeomorphic to $\operatorname{hocolim}_{\mathcal{C}} Y$.

Lemma 1.3 Let $Y$ be a covariant $\mathcal{C}$-space. Then $\mathrm{E}^{\text {bar }} \mathcal{T}_{\mathcal{C}}(Y) \otimes_{\mathcal{T}_{\mathcal{C}}(Y)} *$ is homeomorphic to $\mathrm{E}^{\text {bar }} \mathcal{C} \otimes_{\mathcal{C}} Y$.

Proof By Lemma 1.1, there is a homeomorphism

$$
\mathrm{E}^{\mathrm{bar}} \mathcal{C} \otimes_{\mathcal{C}} \pi_{*}(*) \rightarrow\left(\mathrm{E}^{\mathrm{bar}} \mathcal{C} \circ \pi\right) \otimes_{\mathcal{T}_{\mathcal{C}}(Y)} *
$$

where $\pi: \mathcal{T}_{\mathcal{C}}(Y) \rightarrow \mathcal{C}$ is the projection functor which sends an object $(C, x)$ to $C$. We will show that $\mathrm{E}^{\mathrm{bar}} \mathcal{C} \otimes_{\mathcal{C}} \pi_{*}(*)$ is homeomorphic to $\mathrm{E}^{\mathrm{bar}} \mathcal{C} \otimes_{\mathcal{C}} Y$ and $\left(\mathrm{E}^{\mathrm{bar}} \mathcal{C} \circ \pi\right) \otimes_{\mathcal{T}_{\mathcal{C}}(Y) *}$ is homeomorphic to $\mathrm{E}^{\text {bar }} \mathcal{T}_{\mathcal{C}}(Y) \otimes_{\mathcal{T}_{\mathcal{C}}(Y)} *$.

Let $C$ be an object of $\mathcal{C}$. A point in $\pi_{*}(*)(C)=\operatorname{Mor}_{\mathcal{C}}(\pi(-), C) \otimes_{\mathcal{T}_{\mathcal{C}}(Y)} *$ is represented by a morphism $\psi: \pi(D, x) \rightarrow C$ in $\mathcal{C}$, where $(D, x)$ is an object of $\mathcal{T}_{\mathcal{C}}(Y)$. Define a natural transformation $\beta: \pi_{*}(*) \rightarrow Y$ by $\beta(C)([\psi])=Y(\psi)(x)$. The inverse, $\beta^{-1}: Y \rightarrow \pi_{*}(*)$, is defined by $\beta^{-1}(C)(y)=\left[\operatorname{id}_{C}\right]$, where $y \in Y(C)$ and $\operatorname{id}_{C}: \pi(C, y) \rightarrow C$ is the identity. This induces a homeomorphism $\mathrm{E}^{\text {bar }} \mathcal{C} \otimes_{\mathcal{C}} \pi_{*}(*) \rightarrow$ $\mathrm{E}^{\mathrm{bar}} \mathcal{C} \otimes_{\mathcal{C}} Y$.

Now let $(C, x)$ be an object of $\mathcal{T}_{\mathcal{C}}(Y)$. Then we have $\left(\mathrm{E}^{\text {bar }} \mathcal{C} \circ \pi\right)(C, x)=\mathrm{E}^{\text {bar }} \mathcal{C}(C)=$ $\mathrm{B}^{\text {bar }}(C \downarrow \mathcal{C})$, and $\mathrm{E}^{\text {bar }} \mathcal{T}_{\mathcal{C}}(Y)(C, x)=\mathrm{B}^{\text {bar }}\left((C, x) \downarrow \mathcal{T}_{\mathcal{C}}(Y)\right)$. For each $(C, x)$ there is an isomorphism of categories $F_{(C, x)}: C \downarrow \mathcal{C} \rightarrow(C, x) \downarrow \mathcal{T}_{\mathcal{C}}(Y)$ given by $F_{(C, x)}(f, A)=$ $(f,(A, Y(f)(x)))$, where $f: C \rightarrow A$ in $\mathcal{C}$. If $\phi:(f, A) \rightarrow\left(f^{\prime}, A^{\prime}\right)$ is a morphism in 
$C \downarrow \mathcal{C}$, then $F_{(C, x)}(\phi)=\phi:(f,(A, Y(f)(x))) \rightarrow\left(f^{\prime},\left(A^{\prime}, Y\left(f^{\prime}\right)(x)\right)\right)$ is a morphism in $(C, x) \downarrow \mathcal{T}_{\mathcal{C}}(Y)$, since $f^{\prime}=\phi \circ f$. The inverse of $F$ is the obvious one. Define the natural transformation $\alpha: \mathrm{E}^{\mathrm{bar}} \mathcal{C} \circ \pi \rightarrow \mathrm{E}^{\mathrm{bar}} \mathcal{T}_{\mathcal{C}}(Y)$ by sending $(C, x)$ to $\mathrm{B}^{\text {bar }}\left(F_{(C, x)}\right)$ : $\mathrm{B}^{\text {bar }}(C \downarrow \mathcal{C}) \rightarrow \mathrm{B}^{\text {bar }}\left((C, x) \downarrow \mathcal{T}_{\mathcal{C}}(Y)\right)$, and define its inverse by $\alpha^{-1}(C, x)=\mathrm{B}^{\mathrm{bar}}\left(F_{(C, x)}^{-1}\right)$. This induces a homeomorphism $\left(\mathrm{E}^{\mathrm{bar}} \mathcal{C} \circ \pi\right) \otimes_{\mathcal{T}_{\mathcal{C}}(Y)} * \rightarrow$ $\mathrm{E}^{\mathrm{bar}} \mathcal{T}_{\mathcal{C}}(Y) \otimes_{\mathcal{T}_{\mathcal{C}}(Y)} *$

If $H: \mathcal{D} \rightarrow \mathcal{C}$ is a covariant functor and $Y$ is a covariant $\mathcal{C}$-space, then there is a functor $\hat{H}: \mathcal{T}_{\mathcal{D}}(Y \circ H) \rightarrow \mathcal{T}_{\mathcal{C}}(Y)$ given by $\hat{H}(D, x)=(H(D), x)$. This induces a map $\mathrm{B}^{\text {bar }}(\hat{H}): \mathrm{B}^{\text {bar }} \mathcal{T}_{\mathcal{D}}(Y \circ H) \rightarrow \mathrm{B}^{\text {bar }} \mathcal{T}_{\mathcal{C}}(Y)$. The functor $H$ also induces a map $\bar{H}: \mathrm{E}^{\text {bar }} \mathcal{D} \otimes_{\mathcal{D}} Y \circ H \rightarrow \mathrm{E}^{\text {bar }} \mathcal{C} \otimes_{\mathcal{C}} Y$ given by $\bar{H}([x, y])=\left[\mathrm{B}^{\text {bar }}\left(H_{D}\right)(x), y\right]$, where $x \in \mathrm{B}^{\text {bar }}(D \downarrow \mathcal{D}), y \in Y(H(D))$ and $H_{D}:(D \downarrow \mathcal{D}) \rightarrow(H(D) \downarrow \mathcal{C})$ is the obvious functor induced by $H$. The maps $\mathrm{B}^{\mathrm{bar}}(\hat{H})$ and $\bar{H}$ are equivalent via the homeomorphism from Lemma 1.3. It is also straightforward to check that the composition of the homeomorphism from Lemma 1.3 with $\mathrm{B}^{\text {bar }}(\pi)$ : $\mathrm{B}^{\text {bar }} \mathcal{T}_{\mathcal{C}}(Y) \rightarrow \mathrm{B}^{\text {bar }} \mathcal{C}$ is equal to $\bar{\pi}: \operatorname{hocolim}_{\mathcal{C}} Y \rightarrow \mathrm{B}^{\mathrm{bar}} \mathcal{C}$.

The transport category definition of the homotopy colimit is employed to prove the following useful lemma.

Lemma 1.4 Let $H: \mathcal{D} \rightarrow \mathcal{C}$ be a covariant functor and $Y$ be a covariant $\mathcal{C}$-space. Then

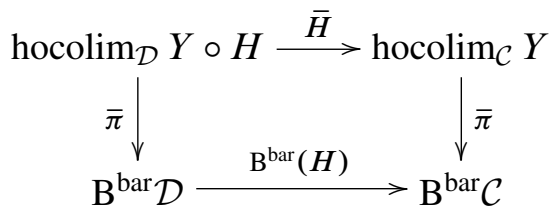

is a pullback diagram.

Proof Form the pullback diagram

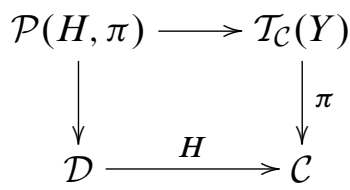

in the category of small categories. The category $\mathcal{P}(H, \pi)$ is a subcategory of $\mathcal{T}_{\mathcal{C}}(Y) \times \mathcal{D}$, where an object $((C, x), D)$ satisfies $H(D)=C$, and a morphism $(\alpha, \beta):((C, x), D) \rightarrow\left(\left(C^{\prime}, x^{\prime}\right), D^{\prime}\right)$ satisfies $\alpha=H(\beta)$. If $((C, x), D)$ is an object of $\mathcal{P}(H, \pi)$, then $(C, x)$ is an object of $\mathcal{T}_{\mathcal{D}}(Y \circ H)$, and if $(\alpha, \beta):((C, x), D) \rightarrow$ $\left(\left(C^{\prime}, x^{\prime}\right), D^{\prime}\right)$ is a morphism of $\mathcal{P}(H, \pi)$, then $\beta:(D, x) \rightarrow\left(D^{\prime}, x^{\prime}\right)$ is a morphism 
of $\mathcal{T}_{\mathcal{D}}(Y \circ H)$, since $(Y \circ H)(\beta)(x)=F(\alpha)(x)=x^{\prime}$. Hence, we have a functor from $\mathcal{P}(H, \pi)$ to the transport category $\mathcal{T}_{\mathcal{D}}(Y \circ H)$ with inverse given by sending $(D, x)$ to $(H(D), x), D)$ and $\left.\beta:(D, x) \rightarrow\left(D^{\prime}, x^{\prime}\right) \mapsto(H(\beta), \beta):(H(D), x), D\right) \rightarrow$ $\left.\left(H\left(D^{\prime}\right), x^{\prime}\right), D^{\prime}\right)$. Therefore, we have the pullback diagram:

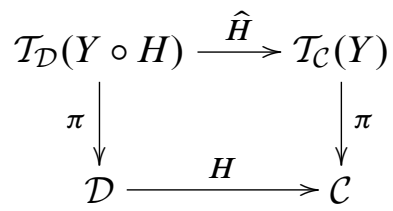

Applying $\mathrm{B}^{\mathrm{bar}}$ produces the pullback diagram:

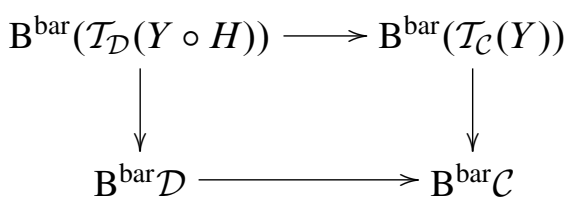

The result now follows from two applications of Lemma 1.3.

\section{The orbit category and the Hochschild complex}

Let $G$ be a discrete group and $\mathcal{F}$ a family of subgroups of $G$ that is closed under conjugation and finite intersections. Let $\mathcal{O}=\operatorname{Or}(G, \mathcal{F})$ denote the orbit category of $G$ with respect to $\mathcal{F}$. The objects of $\mathcal{O}$ are the homogeneous spaces $G / H$, with $H$ in $\mathcal{F}$, considered as left $G$-sets. Morphisms are all $G$-equivariant maps. Therefore, $\operatorname{Mor}_{\mathcal{O}}(G / H, G / K)=\left\{r_{g} \mid g^{-1} H g \leq K\right\}$, where $r_{g}$ is right multiplication by $g$, ie, $r_{g}(u H)=(u g) H$ for $u H$ in $G / H$. If $\mathcal{F}$ is the family of all subgroups of $G$, then $\mathcal{O}$ is called the orbit category. If $\mathcal{F}$ is taken to be the trivial family, then $\mathcal{O}$ is the usual category associated to the group $G$.

Definition 2.1 (Hochschild complex of a group associated to a family of subgroups) Let $\mathcal{O} \times \mathcal{O}$ be the category whose objects are ordered pairs of objects in $\mathcal{O}$ and whose morphisms are ordered pairs of morphisms in $\mathcal{O}$. Let Ad: $\mathcal{O} \times \mathcal{O} \rightarrow \mathrm{CGH}$ be the covariant functor defined by

$$
\begin{aligned}
\operatorname{Ad}\left(G / H_{1}, G / H_{2}\right) & =H_{1} \backslash G / H_{2} \\
\operatorname{Ad}\left(r_{g_{1}}, r_{g_{2}}\right)\left(H_{1} u H_{2}\right) & =K_{1} g_{1}{ }^{-1} u g_{2} K_{2},
\end{aligned}
$$

where $H_{1} \backslash G / H_{2}$ is the set of $\left(H_{1}, H_{2}\right)$ double cosets in $G$ with the discrete topology and $\left(r_{g_{1}}, r_{g_{2}}\right):\left(G / H_{1}, G / H_{2}\right) \rightarrow\left(G / K_{1}, G / K_{2}\right)$ is a morphism in $\mathcal{O} \times \mathcal{O}$. Let $N_{\mathcal{F}}=$ 
Ad $\circ \Delta$, where $\Delta: \mathcal{O} \rightarrow \mathcal{O} \times \mathcal{O}$ is the diagonal functor, and define

$$
\mathfrak{N}(G, \mathcal{F})=\underset{\mathcal{O}}{\operatorname{hocolim}} N_{\mathcal{F}}
$$

We call $\mathfrak{N}(G, \mathcal{F})$ the Hochschild complex of $G$ associated to the family $\mathcal{F}$.

Remark 2.2 More generally, $N_{\mathcal{F}}$ can be defined in the case $G$ is a locally compact topological group and the members of the family of subgroups $\mathcal{F}$ are closed subgroups of $G$ by giving $H_{1} \backslash G / H_{2}$ the quotient topology.

If $\mathcal{F}$ is the trivial family, $\{1\}$, then $\mathfrak{N}(G,\{1\})$ is homotopy equivalent to $\left|N^{\text {cyc }}(G)\right|$, the geometric realization of the cyclic bar construction [12, 7.3.10]; indeed, using the twosided bar construction as a model for the homotopy colimit of $N_{\{1\}}$ yields a complex homeomorphic to $\left|N^{\text {cyc }}(G)\right|$. We refer to $\mathfrak{N}(G,\{1\})$ as the classical Hochschild complex of $G$.

Definition 2.3 The Hochschild homology of a group ring $\mathbb{Z} G$ relative to a family of subgroups $\mathcal{F}$ of $G$ is defined to be

$$
H H_{*}^{\mathcal{F}}(\mathbb{Z} G):=H_{*}(\mathfrak{N}(G, \mathcal{F}) ; \mathbb{Z}) .
$$

Using diagram (1) with Ad and $N_{\mathcal{F}}$, we obtain the following pullback diagram

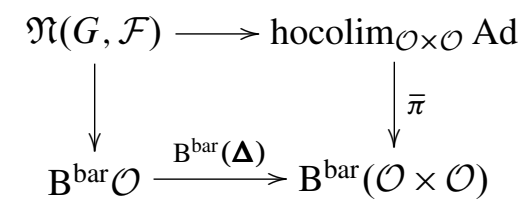

Lemma 2.4 Let $\Delta: \mathcal{O} \rightarrow \mathcal{O} \times \mathcal{O}$ denote the diagonal functor. Then hocolim ${ }_{\mathcal{O} \times \mathcal{O}} \mathrm{Ad}$ is homeomorphic to $\left(\mathrm{E}^{\mathrm{bar}}(\mathcal{O} \times \mathcal{O}) \circ \Delta\right) \otimes_{\mathcal{O}} *$.

Proof Let $T: \mathcal{O} \times \mathcal{O} \rightarrow \mathrm{CGH}$ denote the covariant functor

$$
\operatorname{Mor}_{\mathcal{O} \times \mathcal{O}}(\Delta(-\mathcal{O}),-\mathcal{O} \times \mathcal{O}) \otimes_{\mathcal{O}} *
$$

Note that $\operatorname{Mor}_{\mathcal{O}}(G / L, G / M)=\left\{r_{g} \mid g^{-1} L g \leq M\right\} \cong\left\{g M \mid g^{-1} L g \leq M\right\}$. Using this identification, let $\alpha$ : Ad $\rightarrow T$ be the natural transformation defined by

$$
\alpha(H \backslash G / K)(H g K)=\left[r_{1}, r_{g}\right],
$$

where $\left(r_{1}, r_{g}\right) \in \operatorname{Mor}_{\mathcal{O} \times \mathcal{O}}((G / 1, G / 1),(G / H, G / K))$. The inverse of $\alpha$ is given by

$$
\alpha^{-1}(G / H, G / K)\left(\left[r_{g_{1}}, r_{g_{2}}\right]\right)=H g_{1}^{-1} g_{2} K
$$


where $\left(r_{g_{1}}, r_{g_{2}}\right) \in \operatorname{Mor}_{\mathcal{O} \times \mathcal{O}}((G / L, G / L),(G / H, G / K))$ and $G / L$ is an object in $\mathcal{O}$. Thus, Ad is naturally equivalent to $T$. Therefore,

$$
\mathrm{E}^{\mathrm{bar}}(\mathcal{O} \times \mathcal{O}) \otimes_{\mathcal{O} \times \mathcal{O}} \text { Ad } \stackrel{\alpha *}{\cong} \mathrm{E}^{\mathrm{bar}}(\mathcal{O} \times \mathcal{O}) \otimes_{\mathcal{O} \times \mathcal{O}} T \stackrel{e}{\cong}\left(\mathrm{E}^{\mathrm{bar}}(\mathcal{O} \times \mathcal{O}) \circ \Delta\right) \otimes_{\mathcal{O}} *
$$

where $e$ is the homeomorphism from Lemma 1.1.

Definition 2.5 Let $G$ be a discrete group and $\mathcal{F}$ be a family of subgroups of $G$. A universal space for $G$-actions with isotropy in $\mathcal{F}$ is a $G-\mathrm{CW}$ complex, $\mathrm{E}_{\mathcal{F}} G$, whose isotropy groups belong to $\mathcal{F}$ and for every $H$ in $\mathcal{F}$, the fixed point set $\left(\mathrm{E}_{\mathcal{F}} G\right)^{H}$ is contractible. Such a space is unique up to $G$-equivariant homotopy equivalence [14].

Davis and Lück [7, Lemma 7.6] showed that given any model for $\mathrm{EO}, \mathrm{EO} \otimes_{\mathcal{O}} \nabla$ is a universal $G$-space with isotropy in $\mathcal{F}$, where $\nabla: \mathcal{O} \rightarrow \mathrm{CGH}$ is the covariant functor that sends $G / H$ to itself and $r_{g}: G / H \rightarrow G / K$ to itself.

Theorem 2.6 Let $G$ be a discrete group and $\mathcal{F}$ be a family of subgroups of $G$. Then

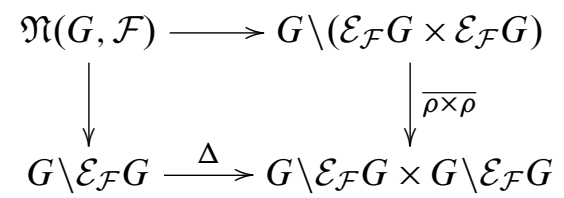

is a pullback diagram, where $\mathcal{E}_{\mathcal{F}} G=\mathrm{E}^{\mathrm{bar}} \mathcal{O} \otimes_{\mathcal{O}} \nabla, \rho: \mathcal{E}_{\mathcal{F}} G \rightarrow G \backslash \mathcal{E}_{\mathcal{F}} G$ is the orbit map, $\overline{\rho \times \rho}$ is the map induced by $\rho \times \rho$, and $\Delta$ is the diagonal map.

Proof There is a homeomorphism

$$
f:\left(\mathrm{E}^{\mathrm{bar}}(\mathcal{O} \times \mathcal{O}) \circ \Delta\right) \otimes_{\mathcal{O}} * \rightarrow G \backslash\left(\mathcal{E}_{\mathcal{F}} G \times \mathcal{E}_{\mathcal{F}} G\right)
$$

defined by $f([(x, y)])=q([x, e K],[y, e K])$, where

$$
(x, y) \in \mathrm{B}^{\mathrm{bar}}(G / K \downarrow \mathcal{O}) \times \mathrm{B}^{\mathrm{bar}}(G / K \downarrow \mathcal{O}) \cong\left(\mathrm{E}^{\mathrm{bar}}(\mathcal{O} \times \mathcal{O}) \circ \Delta\right)(G / K)
$$

and $q: \mathcal{E}_{\mathcal{F}} G \times \mathcal{E}_{\mathcal{F}} G \rightarrow G \backslash\left(\mathcal{E}_{\mathcal{F}} G \times \mathcal{E}_{\mathcal{F}} G\right)$ is the orbit map. The inverse of $f$ is given by $f^{-1}\left(q\left(\left[x, g_{1} K\right],\left[y, g_{2} K\right]\right)\right)=\left[\mathrm{B}^{\mathrm{bar}}\left(\epsilon_{g_{1} K}^{*}\right)(x), \mathrm{B}^{\mathrm{bar}}\left(\epsilon_{g_{2} K}^{*}\right)(y)\right]$, where $\epsilon_{g_{i} K}: G / 1 \rightarrow$ $G / K$ is right multiplication by $g_{i}$. Here we have identified $\mathrm{B}^{\text {bar }}(\mathcal{C} \times \mathcal{D})$ with $\mathrm{B}^{\text {bar }} \mathcal{C} \times$ $\mathrm{B}^{\mathrm{bar}} \mathcal{D}$. Similarly, there is a homeomorphism

$$
\bar{f}: \mathrm{B}^{\text {bar }} \mathcal{O} \cong \mathrm{E}^{\text {bar }} \mathcal{O} \otimes_{\mathcal{O}} * \rightarrow G \backslash \mathcal{E}_{\mathcal{F}} G
$$

defined by $\bar{f}([x])=\rho([x, e K])$, where $x \in \mathrm{B}^{\text {bar }}(G / K \downarrow \mathcal{O})$ and $\rho: \mathcal{E}_{\mathcal{F}} G \rightarrow G \backslash \mathcal{E}_{\mathcal{F}} G$ is the orbit map. The inverse of $\bar{f}$ is given by $(\bar{f})^{-1}(\rho([x, g K]))=\left[\mathrm{B}^{\mathrm{bar}}\left(\epsilon_{g K}^{*}\right)(x)\right]$. 
Using the homeomorphism from Lemma 2.4, we get the commutative diagram

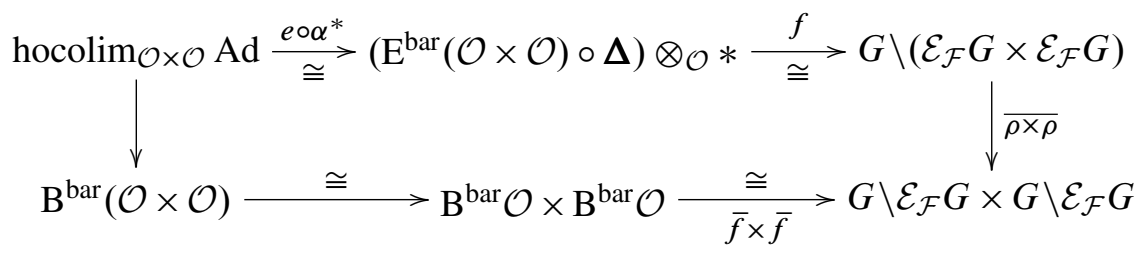

where $(\overline{\rho \times \rho})(q(x, y))=(\rho(x), \rho(y))$. Since $\mathrm{B}^{\text {bar }}(\Delta)$ composed with the homeomorphism $\mathrm{B}^{\text {bar }}(\mathcal{O} \times \mathcal{O}) \rightarrow \mathrm{B}^{\text {bar }} \mathcal{O} \times \mathrm{B}^{\text {bar }} \mathcal{O}$ is just the diagonal map $\Delta: \mathrm{B}^{\text {bar }} \mathcal{O} \rightarrow$ $\mathrm{B}^{\text {bar }} \mathcal{O} \times \mathrm{B}^{\text {bar }} \mathcal{O}$, diagram (2) completes the proof.

Remark 2.7 When $\mathcal{F}$ is the trivial family, the main diagram of Theorem 2.6 becomes:

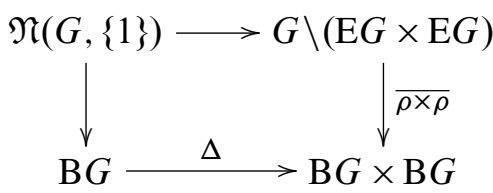

Furthermore, in this case, the map $\overline{\rho \times \rho}$ is a fibration from which it follows that the above square is also a homotopy pullback diagram. This observation is part of the folklore of the subject; indeed, one method of establishing the homotopy equivalence $\left|N^{\text {cyc }}(G)\right| \simeq \mathcal{L}(\mathrm{B} G)$ involves replacing $\overline{\rho \times \rho}$ with the fibration $\mathrm{B} G^{I} \rightarrow \mathrm{B} G \times \mathrm{B} G$, given by evaluation at endpoints where $\mathrm{B} G^{I}$ is the space of paths in $\mathrm{B} G$. For a general family $\mathcal{F}$, Theorem 2.6 is, to our knowledge, new and we note that the map $\overline{\rho \times \rho}$ in Theorem 2.6 is typically not a fibration.

If $\mathcal{F}^{\prime} \subset \mathcal{F}$, then there is an inclusion functor $\iota: \operatorname{Or}\left(G, \mathcal{F}^{\prime}\right) \rightarrow \operatorname{Or}(G, \mathcal{F})$. Clearly, $N_{\mathcal{F}^{\prime}}=N_{\mathcal{F}} \circ \iota$, which induces a map $\mathfrak{N}\left(G, \mathcal{F}^{\prime}\right) \rightarrow \mathfrak{N}(G, \mathcal{F})$. This map is examined in Section 6 in the case when $\mathcal{F}^{\prime}$ is the trivial family and $\mathcal{F}$ is the family of finite subgroups.

\section{The configuration space $F(X)$}

In this section we investigate, in a general context, some basic properties of the configuration space, $F(X)$, of pairs of points in a $G$-space $X$ which lie on the same $G$-orbit.

Let $G$ be a topological group. The category of left $G$-spaces, denoted by ${ }_{G} \mathrm{TOP}$, is the category whose objects are left $G$-spaces, ie, topological spaces $X$ together with a continuous left $G$-action $G \times X \rightarrow X$, written as $(g, x) \mapsto g x$, and whose morphisms 
are continuous equivariant maps $f: X \rightarrow Y$. Henceforth, we abbreviate "left $G$-space" to " $G$-space."

Given a $G$-space $X$, define $A_{X}: G \times X \rightarrow X \times X$ by $A_{X}(g, x):=(x, g x)$ for $(g, x) \in$ $G \times X$. Note that $A_{X}$ is continuous and $G$-equivariant, where $G \times X$ is given the left $G$-action

$$
h(g, x):=\left(h g h^{-1}, h x\right) \text { for } h, g \in G \text { and } x \in X
$$

and $X \times X$ is given the diagonal $G$-action. Hence the image of $A_{X}$ is a $G$-invariant subspace of $X \times X$.

Definition 3.1 Define $F:{ }_{G}$ TOP $\rightarrow_{G}$ TOP on an object $X$ by $F(X):=\operatorname{image}\left(A_{X}\right)$ with the left $G$-action inherited from the diagonal $G$-action on $X \times X$. If $f: X \rightarrow Y$ is equivariant, ie, a morphism in ${ }_{G} \mathrm{TOP}$, then the diagram

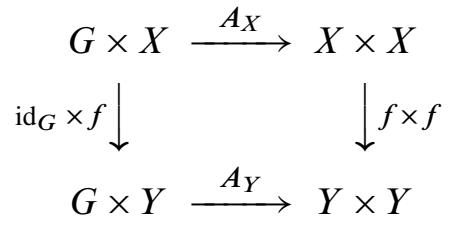

is commutative and so $f \times f$ restricts to an equivariant map $F(f): F(X) \rightarrow F(Y)$. Clearly, $F\left(\operatorname{id}_{X}\right)=\operatorname{id}_{F(X)}$ and $F\left(f_{1} f_{2}\right)=F\left(f_{1}\right) F\left(f_{2}\right)$ for composable morphisms $f_{1}$ and $f_{2}$. That is, $F$ is a functor.

Note that $F(X)$ is the subspace of $X \times X$ consisting of those pairs $(x, y)$ such that $x$ and $y$ lie in the same orbit of the $G$-action.

There is an evident natural isomorphism $F(X) \times I \cong F(X \times I)$, where $I$ is the unit interval with the trivial $G$-action, given by $((x, y), t) \mapsto((x, t),(y, t))$ for $(x, y) \in$ $F(X)$ and $t \in I$. If $H: X \times I \rightarrow Y$ is an equivariant homotopy then

$$
F(X) \times I \stackrel{\cong}{\longrightarrow} F(X \times I) \stackrel{F(H)}{\longrightarrow} Y
$$

is an equivariant homotopy from $F\left(H_{0}\right)$ to $F\left(H_{1}\right)$, where $H_{t}:=H(-, t)$. Hence $F$ factors through the homotopy category of ${ }_{G} \mathrm{TOP}$ with the following consequence.

Proposition 3.2 If the map $f: X \rightarrow Y$ is an equivariant homotopy equivalence, then $F(f): F(X) \rightarrow F(Y)$ is an equivariant homotopy equivalence.

Definition 3.3 In the category TOP of all topological spaces we use the following notation for the standard pullback construction. Given maps $e: A \rightarrow Z$ and $f: B \rightarrow Z$, define $E(e, f):=\{(x, y) \in A \times B \mid e(x)=f(y)\}$ topologized as a subspace of $A \times B$ 
with the product topology. The maps $p_{1}: E(e, f) \rightarrow A$ and $p_{2}: E(e, f) \rightarrow B$ are given, respectively, by the restriction of the projections $A \times B \rightarrow A$ and $A \times B \rightarrow B$. The square

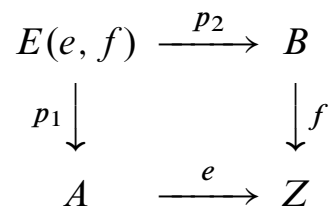

is a pullback diagram in TOP, which we refer to as a standard pullback diagram.

Proposition 3.4 There is a pullback diagram

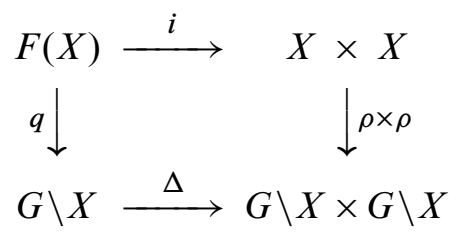

where $i$ is the inclusion $F(X)=\operatorname{image}\left(A_{X}\right) \subset X \times X, \rho: X \rightarrow G \backslash X$ is the orbit map, $\Delta$ is the diagonal map and $q: F(X) \rightarrow G \backslash X$ is given by $q((x, y))=\rho(y)$ for $(x, y) \in F(X)$.

Proof The standard pullback construction yields

$$
E(\Delta, \rho \times \rho)=\left\{\left(\rho(x), x_{1}, x_{2}\right) \in(G \backslash X) \times X \times X \mid \rho(x)=\rho\left(x_{1}\right)=\rho\left(x_{2}\right)\right\} .
$$

The map $j: F(X) \rightarrow E(\Delta, \rho \times \rho)$ given by $j((x, y))=(\rho(x), x, y)$ is a homeomorphism with inverse $(\rho(x), x, y) \mapsto(x, y)$. Also $p_{1} j=q$ and $p_{2} j=i$, where $p_{1}: E(\Delta, \rho \times \rho) \rightarrow G \backslash X$ and $p_{2}: E(\Delta, \rho \times \rho) \rightarrow X \times X$ are the restrictions of the corresponding projections.

The space $G \backslash F(X)$ can also be described as a pullback as follows:

Theorem 3.5 There is a pullback diagram

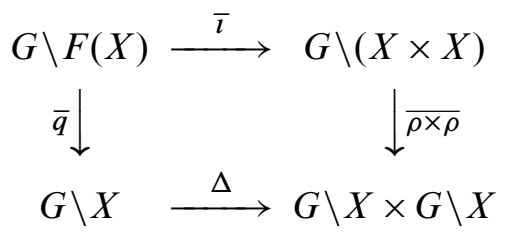

where $\bar{l}, \bar{q}$ and $\overline{\rho \times \rho}$ are induced by $i, q$ and $\rho \times \rho$ respectively (as in Proposition 3.4). 
Proof The pullback diagram of Proposition 3.4 factors as:

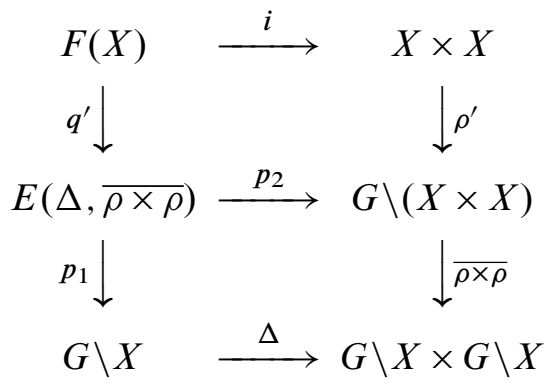

where $\rho^{\prime}: X \times X \rightarrow G \backslash(X \times X)$ is the orbit map, $q^{\prime}((x, y))=\left(\rho(x), \rho^{\prime}(x, y)\right)$ for $(x, y) \in F(X)$ and $E(\Delta, \overline{\rho \times \rho})$ together with the maps $p_{1}, p_{2}$ is the standard pullback construction. The outer square in the above diagram is a pullback by Proposition 3.4 and the lower square is a pullback by construction. It follows that the upper square is a pullback. By Lemma 3.18, $q^{\prime}$ induces a homeomorphism $G \backslash F(X) \cong E(\Delta, \overline{\rho \times \rho})$.

A Hausdorff space $X$ is compactly generated if a set $A \subset X$ is closed if and only if it meets each compact set of $X$ in a closed set.

Proposition 3.6 Suppose that $G$ is a countable discrete group and that $X$ is a countable $G-C W$ complex, ie, $X$ has countably many $G$-cells. Then $F(X)$ and $G \backslash F(X)$ are compactly generated Hausdorff spaces.

Proof Milnor showed that the product of two countable CW complexes is a CW complex [18, Lemma 2.1]. Since $X$ and $G \backslash X$ are countable CW complexes, the product $G \backslash X \times X \times X$ is also a CW complex and thus compactly generated. By Proposition 3.4, $F(X)$ is homeomorphic to a closed subset of this space and hence must be compactly generated. The space $X \times X$ is a CW complex and so $G \backslash(X \times X)$ is also a CW complex because the diagonal $G$-action on $X \times X$ is cellular. By Theorem 3.5, $G \backslash F(X)$ is homeomorphic to a closed subset of the CW complex $G \backslash X \times G \backslash(X \times X)$ and hence must compactly generated.

Recall that for a discrete group $G$ and family of subgroups $\mathcal{F}$, we denote the bar construction model for the universal space for $G$-actions with isotropy in $\mathcal{F}$ by $\mathcal{E}_{\mathcal{F}} G$ (see Theorem 2.6).

Theorem 3.7 Suppose that $G$ is a countable discrete group and that $\mathcal{F}$ is a countable family of subgroups. Then there is a natural homeomorphism $\mathfrak{N}(G, \mathcal{F}) \cong G \backslash F\left(\mathcal{E}_{\mathcal{F}} G\right)$. 
Proof By Theorem 3.5, there is a pullback diagram in TOP:

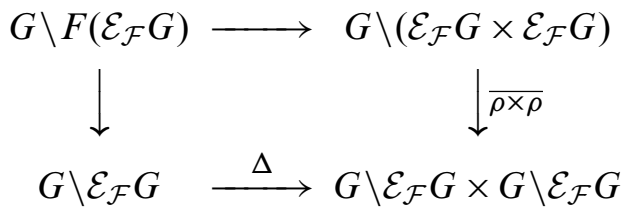

Since $G$ and $\mathcal{F}$ are countable, $\mathcal{E}_{\mathcal{F}} G$ is a countable $\mathrm{CW}$ complex. All the spaces appearing the above diagram are compactly generated by Proposition 3.6 and its proof. It follows that this diagram is also a pullback diagram in the category of compactly generated Hausdorff spaces. A comparison with the pullback diagram in the statement of Theorem 2.6 yields a natural homeomorphism $\mathfrak{N}(G, \mathcal{F}) \cong G \backslash F\left(\mathcal{E}_{\mathcal{F}} G\right)$.

Corollary 3.8 Suppose that $G$ is a countable discrete group and that $\mathcal{F}$ is a countable family of subgroups. Let $\mathrm{E}_{\mathcal{F}} G$ be any $G-C W$ model for the universal space for $G-$ actions with isotropy in $\mathcal{F}$. Then there is a natural homotopy equivalence $\mathfrak{N}(G, \mathcal{F}) \simeq$ $G \backslash F\left(\mathrm{E}_{\mathcal{F}} G\right)$.

Proof There is an equivariant homotopy equivalence $J: \mathcal{E}_{\mathcal{F}} G \rightarrow \mathrm{E}_{\mathcal{F}} G$, which is unique up to equivariant homotopy. By Proposition 3.2, $J$ induces a homotopy equivalence $G \backslash F\left(\mathcal{E}_{\mathcal{F}} G\right) \rightarrow G \backslash F\left(\mathrm{E}_{\mathcal{F}} G\right)$. Composition with the homeomorphism of Theorem 3.7 yields the conclusion.

Note that in Corollary 3.8, "natural" means that for an inclusion $\mathcal{F}^{\prime} \subset \mathcal{F}$ of families of subgroups of $G$, the corresponding square diagram is homotopy commutative.

Recall that a continuous map $f: Y \rightarrow Z$ is proper if for any topological space $W$, $f \times \operatorname{id}_{W}: Y \times W \rightarrow Z \times W$ is a closed map (equivalently, $f$ is a closed map with quasicompact fibers $[4, \mathrm{I}, 10.2$, Theorem 1(b)]). There are several distinct notions of a "proper action" of a topological group on a topological space; see Biller [2] for their comparison. We will use the following definition (see Bourbaki [4, III, 4.1, Definition 1]).

Definition 3.9 A left action of a topological group $G$ on a topological space $X$ is proper provided the map $A_{X}: G \times X \rightarrow X \times X$ is proper, in which case we say that $X$ is a proper $G-$ space.

Proposition 3.10 Suppose that the topological group $G$ acts freely and properly on the $G$-space $X$. Then $A_{X}: G \times X \rightarrow F(X)$ is a homeomorphism. Consequently, $A_{X}$ induces a homeomorphism $\bar{A}_{X}: G \backslash(G \times X) \rightarrow G \backslash F(X)$, where the $G$-action on $G \times X$ is given by Equation (3). 
Proof Clearly $A_{X}$ is a continuous surjection. Since the $G$-action is proper, $A_{X}$ is a closed map. If $A_{X}\left(g_{1}, x_{1}\right)=A_{X}\left(g_{2}, x_{2}\right)$, then $x_{1}=x_{2}$ and $g_{1} x_{1}=g_{2} x_{2}$. Since the $G$-action is free, $g_{1}=g_{2}$ and so $A_{X}$ is injective. Thus, $A_{X}$ is a homeomorphism.

Let $\operatorname{conj}(G)$ denote the set of conjugacy classes of the group $G$. For $g \in G$, let $C(g) \in \operatorname{conj}(G)$ denote the conjugacy class of $g$, and let $Z(g):=\{h \in G \mid h g=g h\}$ denote the centralizer of $g$.

Proposition 3.11 Suppose that $G$ is a discrete group acting on a topological space $X$. Then there is a homeomorphism

$$
G \backslash(G \times X) \cong \coprod_{C(g) \in \operatorname{conj}(G)} Z(g) \backslash X,
$$

where the right side of the isomorphism is a disjoint topological sum.

Proof The space $G \times X$ is the disjoint union of the $G$-invariant subspaces $C(g) \times X$, $C(g) \in \operatorname{conj}(G)$. Since $G$ is discrete, $C(g) \times X$ is both open and closed in $G \times X$. It follows that $G \backslash(G \times X)$ is the disjoint topological sum of the spaces $G \backslash(C(g) \times X)$, $C(g) \in \operatorname{conj}(G)$. The map $G \backslash(C(g) \times X) \rightarrow Z(g) \backslash X$, which takes the $G$-orbit of $\left(h g h^{-1}, x\right)$ to the $Z(g)$-orbit of $h^{-1} x$, is a homeomorphism whose inverse is the map that takes the $Z(g)$-orbit of $x \in X$ to the $G$-orbit of $(g, x)$.

Combining Proposition 3.10 and Proposition 3.11 yields:

Corollary 3.12 Suppose that $G$ is a discrete group that acts freely and properly on a topological space $X$. Then there is a homeomorphism

$$
G \backslash F(X) \cong \coprod_{C(g) \in \operatorname{conj}(G)} Z(g) \backslash X,
$$

where the right side of the isomorphism is a disjoint topological sum.

Remark 3.13 A discrete group $G$ acts freely and properly on a space $X$ if and only if $G \backslash X$ is Hausdorff and the orbit map $\rho: X \rightarrow G \backslash X$ is a covering projection.

As a consequence of Corollary 3.12, if a nontrivial discrete group $G$ acts freely and properly on a nonempty topological space $X$ then $G \backslash F(X)$ is never connected. However, if $G$ acts properly but not freely, then $F(X)$, hence also $G \backslash F(X)$, can be connected (see Example 5.5 and Example 5.6). 
Definition 3.14 Let $X$ be a $G$-space. The subspace $F(X)_{0} \subset F(X)$ is defined to be the union of the connected components of $F(X)$ that meet the diagonal of $X \times X$, ie, the subspace $\Delta(X)=\{(x, x) \in X \times X\}$. In particular, if $X$ is connected, then $F(X)_{0}$ is the connected component of $F(X)$ containing $\Delta(X)$.

Proposition 3.15 $F(X)_{0}$ is a $G$-invariant subspace of $F(X)$.

Proof Let $C$ be a component of $F(X)$ such that $C \cap \Delta(X) \neq \varnothing$. Left translation by $g \in G, L_{g}: F(X) \rightarrow F(X)$, is a homeomorphism and so $L_{g}(C)$ is also a component of $F(X)$. Since $\varnothing \neq L_{g}(C \cap \Delta(X))=L_{g}(C) \cap \Delta(X)$, it follows that $L_{g}(C) \subset F(X)_{0}$.

Remark 3.16 Suppose that the discrete group $G$ acts freely and properly on $X$. Then by Proposition 3.10, the map $A_{X}: G \times X \rightarrow F(X)$ is an equivariant homeomorphism and $F(X)_{0}=A_{X}(\{1\} \times X)=\Delta(X)$.

The remainder of this section is devoted to the proof of various elementary lemmas which have been employed above.

Lemma 3.17 Consider the standard pullback diagram:

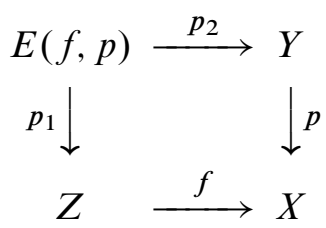

If $p$ is an open map, then $p_{1}$ is also an open map.

Proof Let $V \subset X$ and $W \subset Y$ be open sets. Then $p_{1}((V \times W) \cap E(f, p))=$ $V \cap f^{-1}(p(W))$. Note that $f^{-1}(p(W))$ is open, since the map $p$ is open and $f$ is continuous and so $V \cap f^{-1}(p(W))$ is also open. Since sets of the form $(V \times$ $W) \cap E(f, p)$ give a basis for the topology of $E(f, p)$ and $p_{1}$ preserves unions, the conclusion follows.

Lemma 3.18 Let $G$ be a topological group, let $Y$ be a $G$-space and let $f: Z \rightarrow G \backslash Y$ be a continuous map. Consider the standard pullback diagram:

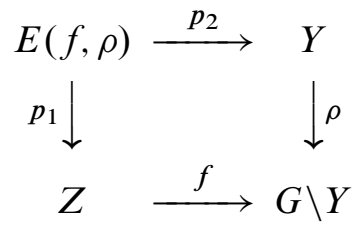

Algebraic 83 Geometric Topology, Volume 8 (2008) 
where $\rho: Y \rightarrow G \backslash Y$ is the orbit map and $G$ acts on $E(f, \rho)$ by $g(z, y):=(z, g y)$ for $g \in G$ and $(z, y) \in E(f, \rho)$. Then $p_{1}$ induces a homeomorphism $\bar{p}_{1}: G \backslash E(f, \rho) \rightarrow Z$ given by $\bar{p}_{1}(q(z, y))=z$ for $(z, y) \in E(f, \rho)$, where $q: E(f, \rho) \rightarrow G \backslash E(f, \rho)$ is the orbit map.

Proof The map $\bar{p}_{1}$ is clearly well-defined and continuous since $p_{1}=\bar{p}_{1} q$ and $G \backslash E(f, \rho)$ has the identification topology determined by the orbit map $q$. Since $\rho$ is surjective, $p_{1}$ is surjective and thus $\bar{p}_{1}$ is also surjective. Suppose $\bar{p}_{1}\left(q\left(z_{1}, x_{1}\right)\right)=$ $\bar{p}_{1}\left(q\left(z_{2}, x_{2}\right)\right)$. Then $z_{1}=z_{2}$ and so $\rho\left(x_{1}\right)=f\left(z_{1}\right)=f\left(z_{2}\right)=\rho\left(x_{2}\right)$. Hence, $q\left(z_{1}, x_{2}\right)=q\left(z_{2}, x_{2}\right)$, demonstrating that $\bar{p}_{1}$ is injective. Since $\rho$ is an open map, $p_{1}$ is also an open map by Lemma 3.17. Let $U \subset G \backslash E(f, \rho)$ be open. Since $q$ is surjective, $U=q\left(q^{-1}(U)\right)$. Thus,

$$
\bar{p}_{1}(U)=\bar{p}_{1} q\left(q^{-1}(U)\right)=p_{1}\left(q^{-1}(U)\right),
$$

which is open since $q^{-1}(U)$ is open and $p_{1}$ is an open map. Therefore, $\bar{p}_{1}$ is an open map. It follows that $\bar{p}_{1}$ is a homeomorphism.

\section{The marked stratified free loop space}

Suppose that $X$ is a proper $G-\mathrm{CW}$ complex, where $G$ is a discrete group. In this section, we show that the orbit space $G \backslash F(X)$ is homotopy equivalent to the space, $P_{\mathrm{sp}}^{\mathrm{m}}(G \backslash X)$, of stratum preserving paths in $G \backslash X$ whose endpoints are "marked" by an orbit of the diagonal action of $G$ on $X \times X$ (see Theorem 4.20). The Covering Homotopy Theorem of Palais plays a key role in the proof of this result. If $X$ satisfies a suitable isovariant homotopy theoretic condition, then $P_{\mathrm{sp}}^{\mathrm{m}}(G \backslash X)$ is shown to be homotopy equivalent to a subspace $\mathcal{L}_{\mathrm{sp}}^{\mathrm{m}}(G \backslash X) \subset P_{\mathrm{sp}}^{\mathrm{m}}(G \backslash X)$, which we call the marked stratified free loop space of $G \backslash X$ (see Theorem 4.23). Applying these results to the case $X=\underline{\mathrm{E}} G$, a universal space for proper $G$-actions, yields a homotopy equivalence between the homotopy colimit, $\mathfrak{N}(G, \mathcal{F})$, of Section 2 and $P_{\mathrm{sp}}^{\mathrm{m}}(G \backslash \underline{\mathrm{E}} G)$ and also, for suitable $G$, to $\mathcal{L}_{\mathrm{sp}}^{\mathrm{m}}(G \backslash X)$ (see Theorem 4.26).

\subsection{Orbit maps as stratified fibrations}

We recall some of the basic definitions from the theory of stratified spaces following the treatment in Hughes [11].

Definition 4.1 A partition of a topological space $X$ consists of an indexing set $\mathcal{J}$ and a collection $\left\{X_{j} \mid j \in \mathcal{J}\right\}$ of pairwise disjoint subspaces of $X$ such that $X=\bigcup_{j \in \mathcal{J}} X_{j}$. For each $j \in \mathcal{J}, X_{j}$ is called the $j$-th stratum. 
A refinement of a partition $\left\{X_{j} \mid j \in \mathcal{J}\right\}$ of a space $X$ is another partition $\left\{X_{i}^{\prime} \mid i \in \mathcal{J}^{\prime}\right\}$ of $X$ such that for every $i \in \mathcal{J}^{\prime}$ there exists $j \in \mathcal{J}$ such that $X_{i}^{\prime} \subset X_{j}$. The component refinement of a partition $\left\{X_{j} \mid j \in \mathcal{J}\right\}$ of $X$ is the refinement obtained by taking the $X_{i}^{\prime}$ 's to be the connected components of the $X_{j}$ 's.

Definition 4.2 A stratification of a topological space $X$ is a locally finite partition $\left\{X_{j} \mid j \in \mathcal{J}\right\}$ of $X$ such that each $X_{j}$ is locally closed in $X$. We say that $X$ together with its stratification is a stratified space.

If $X$ is a space with a given partition, then a map $f: Z \times A \rightarrow X$ is stratum preserving along $A$ if for each $z \in Z, f(\{z\} \times A)$ lies in a single stratum of $X$. In particular, a map $f: Z \times I \rightarrow X$ is a stratum preserving homotopy if it is stratum preserving along $I$.

A class of topological spaces will mean a subclass of the class of all topological spaces, typically defined by a property, for example, the class of all metrizable spaces.

Definition 4.3 Let $X$ and $Y$ be spaces with given partitions. A map $p: X \rightarrow Y$ is a stratified fibration with respect to a class of topological spaces $\mathcal{W}$ if for any space $Z$ in $\mathcal{W}$ and any commutative square

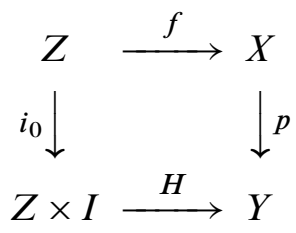

where $i_{0}(z):=(z, 0)$ and $H$ is a stratum preserving homotopy, there exists a stratum preserving homotopy $\widetilde{H}: Z \times I \rightarrow X$ such that $\widetilde{H}(z, 0)=f(z)$ for all $z \in Z$ and $p \widetilde{H}=H$.

Definition 4.4 Let $X$ be a space with a given partition. The space of stratum preserving paths in $X$, denoted by $P_{\mathrm{sp}}(X)$, is the subspace of $X^{I}$, the space of continuous maps of the unit interval into $X$ with the compact-open topology, consisting of stratum preserving paths, ie, paths $\omega: I \rightarrow X$ such that $\omega(I)$ belongs to a single stratum of $X$.

Observe that a homotopy $H: Z \times I \rightarrow X$ is stratum preserving if and only if its adjoint $\hat{H}: Z \rightarrow X^{I}$, given by $\hat{H}(z)(t):=H(z, t)$ for $(z, t) \in Z \times I$, has $\hat{H}(Z) \subset P_{\mathrm{sp}}(X)$.

A group action on a space determines an invariant partition on that space as follows. 
Definition 4.5 (Orbit type partition) Let $G$ be a topological group and let $X$ be a $G$-space. For a subgroup $H \subset G$, let $X_{H}:=\left\{x \in X \mid G_{x}=H\right\}$, where $G_{x}$ is the isotropy subgroup at $x$. Let $(H):=\left\{g H g^{-1} \mid g \in G\right\}$, the set of conjugates of $H$ in $G$, and $X_{(H)}:=\bigcup_{K \in(H)} X_{K}$. Let $\mathcal{J}$ denote the set of conjugacy classes of subgroups of $G$ of the form $\left(G_{X}\right)$. The subspaces $X_{(H)}$ are $G$-invariant and $\left\{X_{(H)} \mid(H) \in \mathcal{J}\right\}$ is a partition of $X$ called the orbit type partition of $X$. Let $\rho: X \rightarrow G \backslash X$ denote the orbit map. The set $\left\{\rho\left(X_{(H)}\right) \mid(H) \in \mathcal{J}\right\}$ is a partition of $G \backslash X$ also called the orbit type partition of $G \backslash X$.

Remark 4.6 If $G$ is a Lie group acting smoothly and properly on a smooth manifold $M$, then the component refinement of the orbit type partition of $M$ is a stratification of $M$, which, in addition, satisfies Whitney's Conditions A and B; see Duistermaat and Kolk [8, Theorem 2.7.4].

An equivariant map $f: X \rightarrow Y$ between two $G$-spaces is isovariant if for every $x \in X$, $G_{x}=G_{f(x)}$. An equivariant homotopy $H: X \times I \rightarrow Y$ is said to be isovariant if for each $t \in I, H_{t}:=H(-, t)$ is isovariant.

We make use of the following version of the Covering Homotopy Theorem of Palais.

Theorem 4.7 (Covering Homotopy Theorem) Let $G$ be a Lie group, let $X$ be a $G$-space and let $Y$ be a proper $G$-space. Assume that every open subset of $G \backslash X$ is paracompact. Suppose that $f: X \rightarrow Y$ is an isovariant map and that $F: G \backslash X \times$ $I \rightarrow G \backslash Y$ is a homotopy such that $F_{0} \circ \rho_{X}=\rho_{Y} \circ f$, where $\rho_{X}: X \rightarrow G \backslash X$ and $\rho_{Y}: Y \rightarrow G \backslash Y$ are the orbit maps, and $F\left(\rho_{X}\left(X_{(H)}\right) \times I\right) \subset \rho_{Y}\left(Y_{(H)}\right)$ for every compact subgroup $H \subset G$. Then there exists an isovariant homotopy $\widetilde{F}: X \times I \rightarrow Y$ such that $\tilde{F}_{0}=f$ and $F \circ\left(\rho_{X} \times \mathrm{id}_{I}\right)=\rho_{Y} \circ \widetilde{F}$.

Remark 4.8 The Covering Homotopy Theorem (CHT) was originally demonstrated by Palais in the case $G$ is a compact Lie group and $X$ and $Y$ are second countable and locally compact $[19,2.4 .1]$. Palais later observed $[20,4.5]$ that his proof of the CHT generalizes to the case of proper actions of a noncompact Lie group. Bredon proved the CHT under the hypotheses that $G$ is compact and that $G \backslash X$ has the property that every open subset is paracompact [5, II, Theorem 7.3]. A topological space is hereditarily paracompact if every subspace is paracompact, equivalently, if every open subspace is paracompact [16, Appendix I, Lemma 8]. The class of hereditarily paracompact spaces includes all metric spaces (since any metric space is paracompact) and all CW complexes [16, II, sec. 4]. The authors of [1] observed that Bredon's proof of [5, II, Theorem 7.1], from which the CHT is deduced, can be adapted to the case of a proper action of a noncompact Lie group; see the discussion following [1, Theorem 1.5]. Also, 
note that it is not necessary to assume that the $G$-action on $X$ is proper because the induced $G$-action on the standard pullback $E\left(F, \rho_{Y}\right)$ is proper by Lemma 4.9 below.

Lemma 4.9 Suppose that $G \times Y \rightarrow Y$ is a proper action of a topological group $G$ on a Hausdorff space $Y$. Let $Z$ be a Hausdorff space and $f: Z \rightarrow G \backslash Y$ a continuous map. Let $\rho: Y \rightarrow G \backslash Y$ denote the orbit map. Then the induced action of $G$ on the standard pullback $E(f, \rho)$ is proper.

Proof By hypothesis, the map $A_{Y}: G \times Y \rightarrow Y \times Y, A_{Y}(g, y)=(y, g y)$, is proper. Since $Z$ is Hausdorff, the diagonal map $\Delta: Z \rightarrow Z \times Z$ is proper. The product of two proper maps is proper and thus $A_{Y} \times \Delta: G \times Y \times Z \rightarrow Y \times Y \times Z \times Z$ is proper. It follows that $A_{Z \times Y}=h_{2} \circ\left(A_{Y} \times \mathrm{id}_{Z}\right) \circ h_{1}: G \times Z \times Y \rightarrow Z \times Y \times Z \times Y$ is proper, where $h_{1}: G \times Z \times Y \rightarrow G \times Y \times Z$ and $h_{2}: Y \times Y \times Z \times Z \rightarrow Z \times Y \times Z \times Y$ are the "interchange" homeomorphisms $h_{1}(g, z, y)=(g, y, z)$ and $h_{2}\left(y_{1}, y_{2}, z_{1}, z_{2}\right)=$ $\left(z_{1}, y_{1}, z_{2}, y_{2}\right)$. Since the action of $G$ on $Y$ is proper, $G \backslash Y$ is Hausdorff [4, III, 4.2, Proposition 3] and so $E(f, \rho)$ is a closed subset of $Z \times Y$. Hence the restriction of $A_{Z \times Y}$ to $G \times E(f, \rho)$ is a proper map. This restriction map factors as $i \circ A_{E(f, \rho)}$ where $i: E(f, \rho) \times E(f, \rho) \hookrightarrow Z \times Y \times Z \times Y$ is inclusion and thus $A_{E(f, \rho)}$ is a proper map ([4, I, 10.2, Proposition 5(d)]).

Theorem 4.10 Suppose that $G$ is a Lie group and that $Y$ is a proper $G$-space. Let $Y$ and $G \backslash Y$ have the orbit type partitions. Then the orbit map $\rho: Y \rightarrow G \backslash Y$ is a stratified fibration with respect to the class of hereditarily paracompact spaces.

Proof Let $Z$ be a hereditarily paracompact space, let $F: Z \times I \rightarrow G \backslash Y$ be a homotopy that is stratum preserving along $I$ and let $f: Z \rightarrow Y$ be a map such that $\rho \circ f=F_{0}$. Consider the standard pullback diagram:

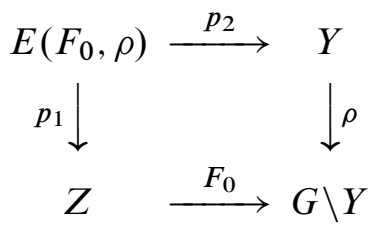

By Lemma 3.18, $p_{1}$ induces a homeomorphism $\bar{p}_{1}: G \backslash E\left(F_{0}, \rho\right) \rightarrow Z$. The map $p_{2}$ is clearly isovariant. The CHT (Theorem 4.7) implies that there is an isovariant homotopy $\widetilde{F}: E\left(F_{0}, \rho\right) \times I \rightarrow Y$ such that $\rho \circ \widetilde{F}=F \circ\left(p_{1} \times \mathrm{id}_{I}\right)$ and $\widetilde{F}_{0}=p_{2}$. Define $\hat{f}: Z \rightarrow E\left(F_{0}, \rho\right)$ by $\hat{f}(z)=(z, f(z))$ for $z \in Z$. Let $\bar{F}: Z \times I \rightarrow Y$ be given by $\bar{F}=\widetilde{F} \circ\left(\hat{f} \times \operatorname{id}_{I}\right)$. Then $\rho \circ \bar{F}=F$ and $\bar{F}_{0}=f$; furthermore, $\bar{F}$ is stratum preserving along $I$. 
Corollary 4.11 Suppose that $G$ is a Lie group and that $Y$ is a proper $G$-space. Let $H \subset G$ be a subgroup. Then the orbit map $\rho: Y_{(H)} \rightarrow G \backslash Y_{(H)}$ is a Serre fibration.

Proof Suppose that $Z$ is a compact polyhedron. Then $Z$ is metrizable and thus hereditarily paracompact. Given a homotopy $F: Z \times I \rightarrow G \backslash Y_{(H)}$ and a map $f: Z \rightarrow Y_{(H)}$ such that $F_{0}=\rho \circ f$, apply Theorem 4.10 to $j \circ F$ and $i \circ f$, where $i: Y_{(H)} \hookrightarrow Y$ and $j: G \backslash Y_{(H)} \hookrightarrow G \backslash Y$ are the inclusions, to obtain $\widetilde{F}: Z \times I \rightarrow Y_{(H)}$ with $\rho \circ \widetilde{F}=F$ and $\widetilde{F}_{0}=f$.

\subsection{Spaces of marked stratum preserving paths}

We apply the results of Section 4.1 in the case $G$ is a discrete group to show that, for a proper $G-\mathrm{CW}$ complex $X$, the orbit space $G \backslash F(X)$ is homotopy equivalent to the space, $P_{\mathrm{sp}}^{\mathrm{m}}(G \backslash X)$, of stratum preserving paths in $G \backslash X$ whose endpoints are "marked" by an orbit of the diagonal action of $G$ on $X \times X$; see Theorem 4.20. That theorem together with Corollary 3.8 and Corollary 4.24 are used to prove Theorem 4.26 , which subsumes Theorem B as stated in the introduction to this paper.

Lemma 4.12 Suppose that $G$ is a discrete group and that $Y$ is a proper $G$-space. Then the orbit map $\rho: Y \rightarrow G \backslash Y$ has the unique path lifting property for stratum preserving paths. That is, given a stratum preserving path $\omega: I \rightarrow G \backslash Y$ and $y \in \rho^{-1}(\omega(0))$ there exists a unique path $\widetilde{\omega}: I \rightarrow Y$ such that $\widetilde{\omega}(0)=y$ and $\rho \circ \widetilde{\omega}=\omega$.

Proof Let $\omega: I \rightarrow G \backslash Y$ be a stratum preserving path, ie, there exists a finite subgroup $H \subset G$ such that $\omega(I) \subset \rho\left(Y_{(H)}\right)=G \backslash Y_{(H)}$. By Corollary 4.11, the restriction of $\rho$ to $Y_{(H)}, \rho: Y_{(H)} \rightarrow G \backslash Y_{(H)}$, is a Serre fibration. The fiber over $\rho(y)$, where $y \in Y_{(H)}$, is the orbit $G \cdot y$, which is discrete since the $G$-action on $Y$ is proper. By [21, 2.2 Theorem 5], a fibration with discrete fibers has the unique path lifting property (note that in the cited theorem, the given fibration is assumed to be a Hurewicz fibration; however, the proof of this theorem uses only the homotopy lifting property respect to $I$ and so remains valid for a Serre fibration).

Combining Theorem 4.10 and Lemma 4.12 yields:

Proposition 4.13 (Unique lifting) Suppose that $G$ is a discrete group and that $Y$ is a proper $G$-space. Let $Z$ be a hereditarily paracompact space. Suppose that $F: Z \times I \rightarrow G \backslash Y$ is stratum preserving homotopy and that $f: Z \rightarrow Y$ is a map such that $\rho \circ f=F_{0}$. Then there exists a unique stratum preserving homotopy $\widetilde{F}: Z \times I \rightarrow Y$ such that $\rho \circ \widetilde{F}=F$ and $\widetilde{F}_{0}=f$. 
We define a "stratified homotopy" version of $F(X)$ as follows.

Definition 4.14 Let $X$ be a $G$-space with its orbit type partition. The $G$-space $F_{\mathrm{sp}}(X)$ is given by:

$$
F_{\mathrm{sp}}(X):=\left\{(\omega, y) \in P_{\mathrm{sp}}(X) \times X \mid \text { there exists } g \in G \text { such that } y=g \omega(1)\right\},
$$

where $G$ acts on $F_{\mathrm{sp}}(X)$ by the restriction of the diagonal action of $G$ on $P_{\mathrm{sp}}(X) \times X$.

Note that there is a pullback diagram

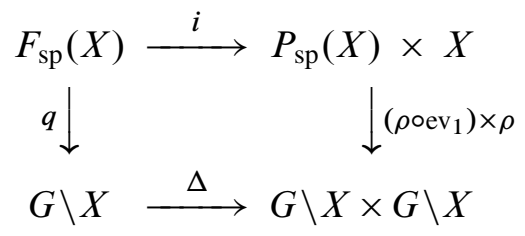

where $i$ is the inclusion $F_{\mathrm{sp}}(X) \hookrightarrow P_{\mathrm{sp}}(X) \times X, \rho: X \rightarrow G \backslash X$ is the orbit map, $\mathrm{ev}_{1}: P_{\mathrm{sp}}(X) \rightarrow X$ is evaluation at $1, \Delta$ is the diagonal map and $q: F_{\mathrm{sp}}(X) \rightarrow G \backslash X$ is given by $q((\omega, y))=\rho(y)$ for $(\omega, y) \in F_{\mathrm{sp}}(X)$.

Proposition 4.15 The map $\ell: F(X) \rightarrow F_{\mathrm{sp}}(X)$ given by $\ell(x, y)=\left(c_{x}, y\right)$, where $c_{x}$ is the constant path at $X$, is an equivariant homotopy equivalence with an equivariant homotopy inverse $j: F_{\mathrm{sp}}(X) \rightarrow F(X)$ given by $j(\omega, y)=(\omega(1), y)$.

Proof Observe that $j \circ \ell=\operatorname{id}_{F(X)}$. Define a homotopy $H: F_{\mathrm{sp}}(X) \times I \rightarrow F_{\mathrm{sp}}(X)$ by $H((\omega, y), t)=\left(\omega_{t}, y\right)$, where $\omega_{t} \in P_{\mathrm{sp}}(X)$ is the path $\omega_{t}(s)=\omega((1-s) t+s)$ for $s \in I$. Then $H$ is an equivariant homotopy from $\operatorname{id}_{F_{\mathrm{sp}}(X)}$ to $\ell \circ j$.

Corollary 4.16 The map $\ell: F(X) \rightarrow F_{\mathrm{sp}}(X)$ induces a homotopy equivalence

$$
\bar{\ell}: G \backslash F(X) \rightarrow G \backslash F_{\mathrm{sp}}(X) .
$$

If $G$ is a Lie group, we say that a $G-\mathrm{CW}$ complex $X$ is proper if $G$ acts properly on $X$. By [13, Theorem 1.23], a $G-\mathrm{CW}$ complex $X$ is proper if and only if for each $x$ in $X$ the isotropy group $G_{X}$ is compact. In particular, if $G$ is discrete, then $X$ is a proper $G-\mathrm{CW}$ complex if and only if $G_{\mathcal{X}}$ is finite for every $x$ in $X$.

Proposition 4.17 Let $G$ be a discrete group. Suppose that $X$ is a proper $G-C W$ complex. Then there is a pullback diagram:

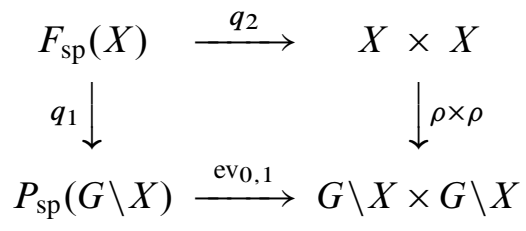


where $\rho: X \rightarrow G \backslash X$ is the orbit map, $q_{1}$ and $q_{2}$ are given, respectively, by $q_{1}(\omega, y)=$ $\rho \circ \omega$ and $q_{2}(\omega, y)=(\omega(0), y)$ for $(\omega, y) \in F_{\mathrm{sp}}(X)$, and $\mathrm{ev}_{0,1}(\tau)=(\tau(0), \tau(1))$ for $\tau \in P_{\mathrm{sp}}(G \backslash X)$.

Proof Let $Z$ be a hereditarily paracompact space. Suppose $h=\left(h_{0}, h_{1}\right): Z \rightarrow X \times X$ and $f: Z \rightarrow P_{\mathrm{sp}}(G \backslash X)$ are maps such that $\mathrm{ev}_{0,1} f=(\rho \times \rho) h$. Let $\check{f}: Z \times I \rightarrow G \backslash X$ be the adjoint of $f$, ie, $\check{f}(z, t)=f(z)(t)$ for $(z, t) \in Z \times I$. Note that $\check{f}$ is stratum preserving along $I$. The diagram

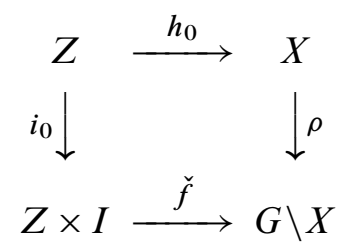

is commutative, where $i_{0}(z)=(z, 0)$ for $z \in Z$. By Proposition 4.13, there exists a unique $F: Z \times I \rightarrow X$ that is stratum preserving along $I$ such that $\rho F=\check{f}$ and $F i_{0}=h_{0}$. Let $\widehat{F}: Z \rightarrow P_{\mathrm{sp}}(X)$ be the adjoint of $F$. Then $Q: Z \rightarrow F_{\mathrm{sp}}(X)$, given by $Q(z)=\left(\widehat{F}(z), h_{1}(z)\right)$ for $z \in Z$, is the unique map such that $h=q_{2} Q$ and $f=q_{1} Q$. In order to conclude that the diagram appearing in the statement of the proposition is a pullback diagram in TOP, it suffices to show that the spaces $F_{\mathrm{sp}}(X)$ and

$$
E\left(\mathrm{ev}_{0,1}, \rho \times \rho\right)=\left\{(\omega, x, y) \in P_{\mathrm{sp}}(G \backslash X) \times X \times X \mid \omega(0)=\rho(x), \omega(1)=\rho(y)\right\}
$$

are hereditarily paracompact. Since $X$ and $G \backslash X$ are CW complexes, the main theorem of [6] implies that the path spaces $X^{I}$ and $(G \backslash X)^{I}$ are stratifiable in the sense of [3, Definition 1.1] (despite the sound alike terminology, this notion of "stratifiable" is not directly related to our Definition 4.2). It is shown in [3] that any CW complex is stratifiable, that a countable product of stratifiable spaces is stratifiable and that a stratifiable space is paracompact and perfectly normal, ie, normal and every closed set is a countable intersection of open sets. Hence $X^{I} \times X$ and $(G \backslash X)^{I} \times X \times X$ are stratifiable and thus paracompact and perfectly normal. A subspace of a paracompact and perfectly normal space is also paracompact and perfectly normal [16, Appendix I, Theorem 10]. In particular, $F_{\mathrm{sp}}(X) \subset X^{I} \times X$ and $E\left(\mathrm{ev}_{0,1}, \rho \times \rho\right) \subset(G \backslash X)^{I} \times X \times X$ and all of their subspaces are paracompact.

Definition 4.18 The space $P_{\mathrm{sp}}^{\mathrm{m}}(G \backslash X)$ of marked stratum preserving paths in $G \backslash X$ consists of stratum preserving paths in $G \backslash X$ whose endpoints are "marked" by an orbit of the diagonal action of $G$ on $X \times X$. More precisely, $P_{\mathrm{sp}}^{\mathrm{m}}(G \backslash X)=E\left(\mathrm{ev}_{0,1}, \overline{\rho \times \rho}\right)$, 
where

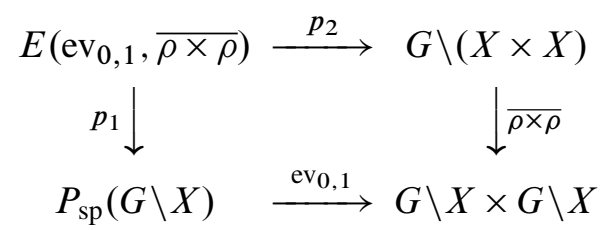

is a standard pullback diagram and $\overline{\rho \times \rho}$ is induced by $\rho \times \rho: X \times X \rightarrow G \backslash X \times G \backslash X$.

Proposition 4.19 Let $G$ be a discrete group. Suppose that $X$ is a proper $G-C W$ complex. Then the map $q: F_{\mathrm{sp}}(X) \rightarrow P_{\mathrm{sp}}^{\mathrm{m}}(G \backslash X)$ given by $q(\omega, y)=\left(\rho \circ \omega, \rho^{\prime}(\omega(0), y)\right)$, where $\rho^{\prime}: X \times X \rightarrow G \backslash(X \times X)$ is the orbit map of the diagonal action, induces a homeomorphism $\bar{q}: G \backslash F_{\mathrm{sp}}(X) \rightarrow P_{\mathrm{sp}}^{\mathrm{m}}(G \backslash X)$.

Proof The pullback diagram of Proposition 4.17 factors as:

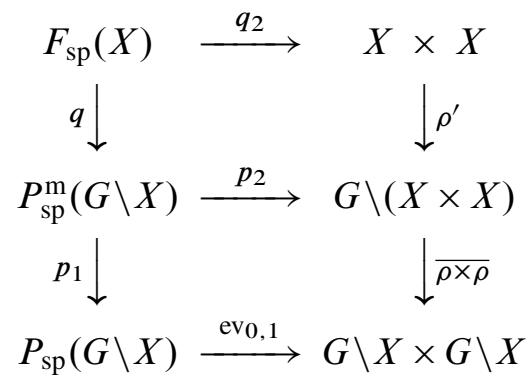

The outer square in the above diagram is a pullback by Proposition 4.17 and the lower square is a pullback by definition. It follows that the upper square is a pullback. By Lemma 3.18, $q$ induces a homeomorphism $\bar{q}: G \backslash F_{\mathrm{sp}}(X) \rightarrow P_{\mathrm{sp}}^{\mathrm{m}}(G \backslash X)$.

Combining Corollary 4.16 and Proposition 4.19 yields:

Theorem 4.20 Let $G$ be a discrete group. Suppose that $X$ is a proper $G-C W$ complex. Then the map $\bar{q} \circ \bar{\ell}: G \backslash F(X) \rightarrow P_{\mathrm{sp}}^{\mathrm{m}}(G \backslash X)$ is a homotopy equivalence.

Definition 4.21 The stratified free loop space of $G \backslash X$, denoted by $\mathcal{L}_{\text {sp }}(G \backslash X)$, is the subspace of $P_{\mathrm{sp}}(G \backslash X)$ consisting of closed paths, ie, $\omega \in P_{\mathrm{sp}}(G \backslash X)$ such that $\omega(0)=\omega(1)$. The marked stratified free loop space of $G \backslash X$, denoted by $\mathcal{L}_{\mathrm{sp}}^{\mathrm{m}}(G \backslash X)$, is the subspace of $P_{\mathrm{sp}}^{\mathrm{m}}(G \backslash X)$ given by:

$$
\mathcal{L}_{\mathrm{sp}}^{\mathrm{m}}(G \backslash X)=\left\{\left(\omega, \rho^{\prime}(x, y)\right) \in P_{\mathrm{sp}}^{\mathrm{m}}(G \backslash X) \mid(x, y) \in F(X)_{0}\right\} .
$$

(Recall that $\rho: X \rightarrow G \backslash X$ and $\rho^{\prime}: X \times X \rightarrow G \backslash(X \times X)$ are the orbit maps and that $F(X)_{0}$ is the union of the components of $F(X)$ meeting the diagonal.) Note that if $\left(\omega, \rho^{\prime}(x, y)\right) \in \mathcal{L}_{\mathrm{sp}}^{\mathrm{m}}(G \backslash X)$, then $\omega(0)=\rho(x)=\rho(y)=\omega(1)$ and so $\omega \in \mathcal{L}_{\mathrm{sp}}(G \backslash X)$. 
There is a standard pullback diagram:

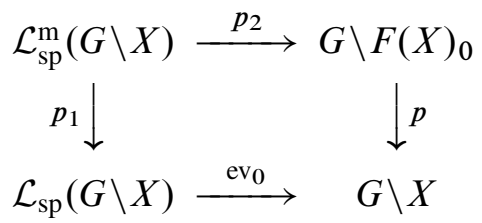

where $p$ is given by $p\left(\rho^{\prime}(x, y)\right)=\rho(x)$ for $\rho^{\prime}(x, y) \in G \backslash F(X)_{0}$.

Let $\bar{\Delta}: G \backslash X \rightarrow G \backslash(X \times X)$ denote the map induced by the diagonal map, $\Delta: X \rightarrow X \times$ $X$. Define the map $\iota: \mathcal{L}_{\mathrm{sp}}(G \backslash X) \rightarrow \mathcal{L}_{\mathrm{sp}}^{\mathrm{m}}(G \backslash X)$ by $\iota(\omega)=(\omega, \bar{\Delta}(\omega(0)))$. The composite $p_{1} \iota$ is the identity map of $\mathcal{L}_{\text {sp }}(G \backslash X)$ and so $\mathcal{L}_{\text {sp }}(G \backslash X)$ is homeomorphic to a retract of $\mathcal{L}_{\mathrm{sp}}^{\mathrm{m}}(G \backslash X)$. In general, $\iota$ is not a homotopy equivalence; for example, in the case of the infinite dihedral group, $D_{\infty}$, acting on $\mathbb{R}$ as in Example 5.5, $\mathcal{L}_{\mathrm{sp}}\left(D_{\infty} \backslash \mathbb{R}\right)$ is contractible, whereas $\mathcal{L}_{\text {sp }}^{\mathrm{m}}\left(D_{\infty} \backslash \mathbb{R}\right)$ is not simply connected.

Proposition 4.22 If the discrete group $G$ acts freely and properly on $X$, then the map $\iota: \mathcal{L}_{\mathrm{sp}}(G \backslash X) \rightarrow \mathcal{L}_{\mathrm{sp}}^{\mathrm{m}}(G \backslash X)$ is a homeomorphism; furthermore, $\mathcal{L}_{\mathrm{sp}}(G \backslash X)=\mathcal{L}(G \backslash X)$, the space of closed paths in $G \backslash X$.

Proof Since the $G$-action on $X$ is free and proper, by Remark 3.16, $F(X)_{0}$ is the diagonal of $X \times X$ and so $p: G \backslash F(X)_{0} \rightarrow G \backslash X$ is a homeomorphism. Thus, $p_{1}: \mathcal{L}_{\mathrm{sp}}^{\mathrm{m}}(G \backslash X) \rightarrow \mathcal{L}_{\text {sp }}(G \backslash X)$ is also homeomorphism, since it is a pullback of $p$. Hence, $\iota=\left(p_{1}\right)^{-1}$ is a homeomorphism. Since the $G$-action is free, there is only one stratum and so $\mathcal{L}_{\text {sp }}(G \backslash X)=\mathcal{L}(G \backslash X)$.

Define $\tilde{S}$ to be the image of the map $G \times P_{\mathrm{sp}}(X) \rightarrow X \times X$ given by $(g, \sigma) \mapsto$ $(\sigma(0), g \sigma(1))$. Note that $\widetilde{S}$ is a $G$-invariant subset of $X \times X$ and that $F(X) \subset \widetilde{S}$.

Theorem 4.23 Suppose that the pair $\left(\tilde{S}, F(X)_{0}\right)$ can be deformed isovariantly into the pair $\left(F(X)_{0}, F(X)_{0}\right)$, ie, there is an isovariant homotopy $H: \widetilde{S} \times I \rightarrow \widetilde{S}$ such that $H(-, 0)$ is the identity of $\widetilde{S}$ and $H\left(\widetilde{S} \times\{1\} \cup F(X)_{0} \times I\right) \subset F(X)_{0}$. Then the inclusion $i: \mathcal{L}_{\mathrm{sp}}^{\mathrm{m}}(G \backslash X) \hookrightarrow P_{\mathrm{sp}}^{\mathrm{m}}(G \backslash X)$ is a homotopy equivalence.

Proof Let $H: \widetilde{S} \times I \rightarrow \widetilde{S}$ be an isovariant homotopy such that $H(-, 0)$ is the identity of $\widetilde{S}$ and $H\left(\widetilde{S} \times\{1\} \cup F(X)_{0} \times I\right) \subset F(X)_{0}$. Write $H=\left(H_{1}, H_{2}\right)$, where $H_{j}: \widetilde{S} \times I \rightarrow X$ for $j=1,2$. Define the homotopy $b: P_{\mathrm{sp}}^{\mathrm{m}}(G \backslash X) \times I \rightarrow P_{\mathrm{sp}}(G \backslash X)$ by

$$
b\left(\left(\omega, \rho^{\prime}(x, y)\right), s\right)(t)= \begin{cases}\rho \circ H_{1}((x, y), s-3 t) & \text { if } 0 \leq t \leq s / 3 \\ \omega((3 t-s) /(3-2 s)) & \text { if } s / 3 \leq t \leq 1-s / 3 \\ \rho \circ H_{2}((x, y), s+3 t-3) & \text { if } 1-s / 3 \leq t \leq 1\end{cases}
$$


where $\rho: X \rightarrow G \backslash X$ and $\rho^{\prime}: X \times X \rightarrow G \backslash(X \times X)$ are the orbit maps. Define the homotopy $B: P_{\mathrm{sp}}^{\mathrm{m}}(G \backslash X) \times I \rightarrow P_{\mathrm{sp}}^{\mathrm{m}}(G \backslash X)$ by

$$
B\left(\left(\omega, \rho^{\prime}(x, y)\right), s\right)=\left(b\left(\left(\omega, \rho^{\prime}(x, y)\right), s\right), \rho^{\prime}(H((x, y), s))\right) .
$$

The hypotheses on $H$ imply that $B$ is a deformation of the pair $\left(P_{\mathrm{sp}}^{\mathrm{m}}(G \backslash X), \mathcal{L}_{\mathrm{sp}}^{\mathrm{m}}(G \backslash X)\right)$ into the pair $\left(\mathcal{L}_{\mathrm{sp}}^{\mathrm{m}}(G \backslash X), \mathcal{L}_{\mathrm{sp}}^{\mathrm{m}}(G \backslash X)\right)$ and so $i: \mathcal{L}_{\mathrm{sp}}^{\mathrm{m}}(G \backslash X) \hookrightarrow P_{\mathrm{sp}}^{\mathrm{m}}(G \backslash X)$ is a homotopy equivalence.

The inclusion $F(X)_{0} \hookrightarrow \widetilde{S}$ is an isovariant strong deformation retract if there is a homotopy $H: \widetilde{S} \times I \rightarrow \widetilde{S}$ as in Theorem 4.23 with the additional property that $H$ is stationary along $F(X)_{0}$.

Corollary 4.24 If $F(X)_{0} \hookrightarrow \widetilde{S}$ is an isovariant strong deformation retract then $i: \mathcal{L}_{\mathrm{sp}}^{\mathrm{m}}(G \backslash X) \hookrightarrow P_{\mathrm{sp}}^{\mathrm{m}}(G \backslash X)$ is a homotopy equivalence.

Remark 4.25 Suppose in Theorem 4.23 that the discrete group $G$ acts freely and properly. Then $\widetilde{S}=X \times X$ and $F(X)_{0}=\Delta(X)$, the diagonal of $X \times X$; see Remark 3.16. The hypothesis of Theorem 4.23 asserts that $(X \times X, \Delta(X))$ is deformable into $(\Delta(X), \Delta(X))$ and so the diagonal map $\Delta: X \rightarrow X \times X$ is a homotopy equivalence. This implies that $X$ is contractible and hence a model for the universal space, $\mathrm{E} G$, for free $G$-actions, provided $X$ has the equivariant homotopy type of a $G-\mathrm{CW}$ complex. Conversely, suppose that $\mathrm{E} G$ is a $G-\mathrm{CW}$ model for the universal space such that $\mathrm{E} G \times \mathrm{E} G$ with the product topology and the diagonal $G$-action is also a $G-\mathrm{CW}$ complex and has an equivariant subdivision such that $\Delta(\mathrm{E} G)$ is a subcomplex. Then $\Delta(\mathrm{E} G) \subset \mathrm{E} G \times \mathrm{E} G$ is an equivariant, hence isovariant (since the $G$-action is free), strong deformation retract.

In Section 5 we show that the hypothesis of Corollary 4.24 is satisfied for a class of groups, which includes the infinite dihedral group and hyperbolic or Euclidean triangle groups, and where $X$ is a universal space for $G$-actions with finite isotropy.

Theorem 4.26 Suppose that $G$ is a countable discrete group and that $\mathcal{F}$ is its family of finite subgroups. Let $\underline{\mathrm{E}} G:=\mathrm{E}_{\mathcal{F}} G$, a universal space for proper $G$-actions, and $\underline{\mathrm{B}} G:=G \backslash \underline{\mathrm{E}} G$.

(i) There is a homotopy equivalence $\mathfrak{N}(G, \mathcal{F}) \simeq P_{\mathrm{sp}}^{\mathrm{m}}(\underline{\mathrm{B}} G)$.

(ii) If $\underline{E} G$ satisfies the hypothesis of Corollary 4.24, then there is a homotopy equivalence $\mathfrak{N}(G, \mathcal{F}) \simeq \mathcal{L}_{\mathrm{sp}}^{\mathrm{m}}(\underline{\mathrm{B}} G)$. 
Proof Conclusion (i) of the theorem is a direct consequence of Corollary 3.8 and Theorem 4.20. Conclusion (ii) follows from (i) and Corollary 4.24.

If $G$ is torsion free, then the family $\mathcal{F}$ of finite subgroups of $G$ is the trivial family and so $\left|N^{\text {cyc }}(G)\right| \simeq \mathfrak{N}(G, \mathcal{F})$ and $\mathcal{L}_{\mathrm{sp}}^{\mathrm{m}}(\underline{\mathrm{B}} G) \cong \mathcal{L}(\mathrm{B} G)$ (Proposition 4.22); furthermore, by Remark 4.25, Theorem 4.26(ii) applies, thereby recovering the familiar result $\left|N^{\text {cyc }}(G)\right| \simeq \mathcal{L}(\mathrm{B} G)$.

\section{Examples}

Let $\underline{\mathrm{E}} G$ denote the universal space for proper $G$-actions and $\underline{\mathrm{B}} G=G \backslash \underline{\mathrm{E}} G$. In this section, we show that if $G$ is the infinite dihedral group or a hyperbolic or Euclidean triangle group, then the hypothesis of Corollary 4.24 is satisfied; that is, $F(\underline{\mathrm{E} G})_{0} \hookrightarrow$ $\widetilde{S}$ is an isovariant strong deformation retract. By Theorem 4.26, this implies that $\mathfrak{N}(G, \mathcal{F}) \simeq P_{\mathrm{sp}}^{\mathrm{m}}(\underline{\mathrm{B}} G) \simeq \mathcal{L}_{\mathrm{sp}}^{\mathrm{m}}(\underline{\mathrm{B}} G)$, where $\mathcal{F}$ is the family of finite subgroups of $G$. This is accomplished by showing that, for these groups, $F(\underline{E} G)$ is path connected and $F(X) \hookrightarrow \widetilde{S}$ is a $G \times G$-isovariant strong deformation retract.

Let $G$ be a discrete group and $X$ a proper $G$-space. Recall that $F(X)$ is the image of $A_{X}: G \times X \rightarrow X \times X$, where $A_{X}(g, x):=(x, g x)$ for $(g, x) \in G \times X$, and $\widetilde{S}$ is the image of the map $G \times P_{\mathrm{sp}}(X) \rightarrow X \times X$ given by $(g, \sigma) \mapsto(\sigma(0), g \sigma(1))$. Notice that $F(X)$ and $\widetilde{S}$ are each $G \times G$-invariant subsets of $X \times X$. Let $\rho: X \rightarrow G \backslash X$ denote the orbit map. Then $F(X)=(\rho \times \rho)^{-1}(\Delta(G \backslash X))$, and $\widetilde{S}=(\rho \times \rho)^{-1}(\{(\sigma(0), \sigma(1)) \mid \sigma \in$ $\left.\left.P_{\mathrm{sp}}(G \backslash X)\right\}\right)$ by Lemma 4.12.

Proposition 5.1 Let $G$ be a discrete group and $X$ a proper $G$-space. Assume that $G \backslash X$ is homeomorphic to a subset of $\mathbb{R}^{n}$ for some $n$, and that the images of the strata of $G \backslash X$ in $\mathbb{R}^{n}$ are convex. Then $F(X) \hookrightarrow \widetilde{S}$ is a $G \times G$-isovariant strong deformation retract.

Proof Let $h$ be a homeomorphism from $G \backslash X$ to $D \subset \mathbb{R}^{n}$ such that the images of the strata of $G \backslash X$ under $h$ are convex. Define $H^{\prime}: \mathbb{R}^{n} \times \mathbb{R}^{n} \times I \rightarrow \mathbb{R}^{n} \times \mathbb{R}^{n}$ by $H^{\prime}((a, b), t)=(a, t a+(1-t) b)$. Notice that $H^{\prime}(a, a, t)=(a, a)$ for every $a \in \mathbb{R}^{n}$ and every $t \in I$. Let $S=\left\{(\sigma(0), \sigma(1)) \mid \sigma \in P_{\mathrm{sp}}(G \backslash X)\right\}$, and let $H=(h \times h)^{-1} \circ$ $H^{\prime} \circ\left(\left.(h \times h)\right|_{S} \times \mathrm{id}_{I}\right)$. Since the images of the strata of $G \backslash X$ under $h$ are convex, $H: S \times I \rightarrow S$ is a homotopy such that $H_{0} \circ(\rho \times \rho)|\tilde{S}=(\rho \times \rho)| \tilde{S} \circ \mathrm{id} \tilde{S}$ and $H\left((\rho \times \rho)\left(\widetilde{S}_{\left(K \times K^{g}\right)}\right) \times I\right) \subset(\rho \times \rho)\left(\widetilde{S}_{\left(K \times K^{g}\right)}\right)$ for every finite subgroup $K$ of $G$ and every $g \in G$. Observe that if $(x, y) \in \widetilde{S}$, then $(G \times G)_{(x, y)}=G_{x} \times G_{y}=K \times K^{g}$ for some finite subgroup $K$ of $G$ and some $g \in G$. Therefore, by the Covering Homotopy 
Theorem (Theorem 4.7), there exists a $G \times G$-isovariant homotopy $\tilde{H}$ : $\widetilde{S} \times I \rightarrow \widetilde{S}$ covering $H$ such that $\widetilde{H}_{0}=\operatorname{id} \tilde{S}$. Since $(\rho \times \rho)^{-1}(\Delta(G \backslash X))=F(X)$, it follows that $\widetilde{H}_{1}(\tilde{S}) \subset F(X)$. Thus, $\widetilde{H}$ is the desired homotopy.

Corollary 5.2 Let $G$ be a discrete group and $X$ a proper $G$-space. Assume that $G \backslash X$ is homeomorphic to a subset of $\mathbb{R}^{n}$ for some $n$, and that the images of the strata of $G \backslash X$ in $\mathbb{R}^{n}$ are convex. If $F(X)$ is path connected, then $F(X)_{0}=F(X) \hookrightarrow \widetilde{S}$ is an isovariant strong deformation retract.

Next we determine when $F(X)$ is path connected.

Theorem 5.3 Let $G$ be a discrete group and $X$ a path connected $G$-space. Then, $F(X)$ is path connected if every element of $G$ can be expressed as a product of elements each of which fixes some point in $X$. If, in addition, $G$ acts properly on $X$, then the converse is true.

Proof Let $S=\{s \in G \mid s y=y$ for some $y \in X\}$. Clearly, if $s \in S$ and $y \in X$ such that $s y=y$, then $A_{X}(s, y)=A_{X}(1, y)$. Since $X$ is path connected, this implies that $A_{X}(S \times X) \subset F(X)$ is path connected.

Suppose $S$ generates $G$. Let $(g, x) \in G \times X$ be given. We will show that there is a path in $F(X)$ connecting $A_{X}(g, x)$ to a point in $A_{X}(S \times X)$. Write $g=s_{n} \cdots s_{2} s_{1}$, where $s_{i} \in S$. For each $i$, there is an $x_{i} \in X$ such that $s_{i} x_{i}=x_{i}$. Therefore,

$A_{X}\left(g, x_{1}\right)=A_{X}\left(g s_{1}^{-1}, x_{1}\right)$ and $A_{X}\left(g s_{1}^{-1} \cdots s_{i}^{-1}, x_{i+1}\right)=A_{X}\left(g s_{1}^{-1} \cdots s_{i+1}^{-1}, x_{i+1}\right)$

for each $i, 1 \leq i \leq n-1$. Since $X$ is path connected, $A_{X}(\{h\} \times X)$ is path connected for every $h \in G$. Thus, $A_{X}(g, x)$ and $A_{X}\left(1, x_{n}\right)$ are connected by a path in $F(X)$.

Now assume that $G$ acts properly on $X$ and that $F(X)$ is path connected. Let $N$ be the subgroup of $G$ generated by $S$. Since $S$ is closed under conjugation, $N$ is a normal subgroup of $G$. Therefore, $G / N$ acts on $N \backslash X$ by $g N \cdot \rho(x)=\rho(g x)$, where $\rho: X \rightarrow N \backslash X$ is the orbit map. It is easy to check that the action is free. The fact that $G$ acts properly on $X$ implies that $N$ acts properly on $X$ and that $X$ is Hausdorff; furthermore, $N \backslash X$ is Hausdorff [4, III, 4.2, Proposition 3]. Recall that a discrete group $G$ acts properly on a Hausdorff space $X$ if and only if for every pair of points $x, y \in X$, there is a neighborhood $V_{x}$ of $x$ and a neighborhood $V_{y}$ of $y$ such that the set of all $g \in G$ for which $g V_{x} \cap V_{y} \neq \varnothing$ is finite [4, III, 4.4, Proposition 7]. This implies that $G / N$ acts properly on $N \backslash X$. Therefore, $A_{G / N}: G / N \times N \backslash X \rightarrow N \backslash X \times N \backslash X$ is a homeomorphism onto its image, $F(N \backslash X)$. Thus, $F(N \backslash X)$ is path connected if and only if $G / N$ is trivial. Since the map $\rho_{F}: F(X) \rightarrow F(N \backslash X)$, defined by $\rho_{F}(x, g x)=(\rho(x), \rho(g x))$, is onto and $F(X)$ is path connected, it follows that $G / N$ is trivial. That is, $G=N$. 
An immediate consequence of this theorem is the following.

Corollary 5.4 Let $G$ be a discrete group and $\mathcal{F}$ a family of subgroups of $G$. If there exists a set of generators, $S$ of $G$, with the property that for every $s \in S$, there is an $H \in \mathcal{F}$ such that $s \in H$, then $F\left(\mathrm{E}_{\mathcal{F}} G\right)$ is path connected.

Example 5.5 (The infinite dihedral group) Let $G=D_{\infty}=\langle a, b| a^{2}=1, a b a^{-1}=$ $\left.b^{-1}\right\rangle$ and $X=\mathbb{R}$, where $a$ acts by reflection through zero and $b$ acts by translation by 1 . Since $\mathbb{R}$ is a model for $\underline{E} D_{\infty}$ and $D_{\infty}$ is generated by two elements of order two, namely $a$ and $a b, F(\mathbb{R})$ is path connected by Corollary 5.4. The quotient of $\mathbb{R}$ by $D_{\infty}$ is homeomorphic to the closed interval $[0,1 / 2]$. The strata are $\{0\},\{1 / 2\}$ and $(0,1 / 2)$. Therefore, Corollary 5.2 implies that $F_{0}(\mathbb{R}) \hookrightarrow \widetilde{S}$ is an isovariant strong deformation retract.

Example 5.6 (Triangle groups) Let

$$
G=\left\langle a, b, c \mid a^{2}=b^{2}=c^{2}=(a b)^{p}=(b c)^{q}=(c a)^{r}=1\right\rangle,
$$

where $p, q, r$ are natural numbers such that $1 / p+1 / q+1 / r \leq 1$. The group $G$ can be realized as a group of reflections through the sides of a Euclidean or hyperbolic triangle whose interior angles measure $\pi / p, \pi / q$ and $\pi / r$, where the generators $a, b$ and $c$ act by reflections through the corresponding sides. Thus, the triangle group $G$ produces a tessellation of the Euclidean or hyperbolic plane by these triangles. Therefore, this plane is a model for $\mathrm{E} G$, whose quotient, $D$, is equivalent to the given triangle. By Corollary 5.4, $F(\underline{E} G)$ is path connected. There are seven strata of $D$, namely $\stackrel{\circ}{D}, S_{a}$, $\stackrel{\circ}{S}_{b}, \stackrel{\circ}{S}_{c}$, and each of the three vertices, where $\stackrel{\circ}{D}$ denotes the interior of $D$, and $\stackrel{\circ}{S}_{a}$, $S_{b}$, and $\dot{S}_{c}$ are the interiors of the sides of the triangle, $S_{a}, S_{b}$, and $S_{c}$, respectively, through which $a, b$, and $c$ reflect. It follows from Corollary 5.2 that $F_{0}(\underline{E} G) \hookrightarrow \widetilde{S}$ is an isovariant strong deformation retract.

Remark 5.7 Let $X$ be a $G$-space and $Y$ an $H$-space. Clearly, $F_{G \times H}(X \times Y) \cong$ $F_{G}(X) \times F_{H}(Y)$ and $F_{G \times H}(X \times Y)_{0} \cong F_{G}(X)_{0} \times F_{H}(Y)_{0}$. (Here, the group that is acting has been added to the notation of the configuration space.) Furthermore, since $(x, y)$ and $\left(x^{\prime}, y^{\prime}\right)$ are in the same stratum of $X \times Y$ if and only if $x$ and $x^{\prime}$ are in the same stratum of $X$ and $y$ and $y^{\prime}$ are in the same stratum of $Y$, it follows that $\widetilde{S}_{X \times Y} \cong \widetilde{S}_{X} \times \widetilde{S}_{Y}$. Therefore, if $F_{G}(X)_{0} \hookrightarrow \widetilde{S}_{X}$ is a $G$-isovariant strong deformation retraction and $F_{H}(Y)_{0} \hookrightarrow \widetilde{S}_{Y}$ is an $H$-isovariant strong deformation retraction, then $F_{G \times H}(X \times Y)_{0} \hookrightarrow \widetilde{S}_{X \times Y}$ is a $G \times H$-isovariant strong deformation retraction. This observation produces interesting examples for which Theorem 4.26 is true. If $X=\mathbb{R}$, $G=\mathbb{Z}, Y=\mathbb{R}$ and $H=D_{\infty}$, then $F_{\mathbb{Z} \times D_{\infty}}(\mathbb{R} \times \mathbb{R}) \cong F_{\mathbb{Z}}(\mathbb{R}) \times F_{D_{\infty}}(\mathbb{R})$ is not path 
connected, since $F_{\mathbb{Z}}(\mathbb{R})$ is not path connected. Moreover, $F_{\mathbb{Z} \times D_{\infty}}(\mathbb{R} \times \mathbb{R})_{0} \neq \Delta(\mathbb{R})$ and $F_{\mathbb{Z} \times D_{\infty}}(\mathbb{R} \times \mathbb{R})_{0} \neq F_{\mathbb{Z} \times D_{\infty}}(\mathbb{R} \times \mathbb{R})$. Despite this, Theorem 4.26 applies to $\mathbb{Z} \times D_{\infty}$.

\section{A comparison of $G \backslash F(E G)$ and $G \backslash F(\underline{E} G)$}

In this section we examine the map $\mathfrak{N}(G,\{1\}) \rightarrow \mathfrak{N}(G, \mathcal{F})$, where $G$ is a discrete group and $\mathcal{F}$ is the family of finite subgroups of $G$. This enables us to compute the induced map $H H_{*}(\mathbb{Z} G) \rightarrow H H_{*}^{\mathcal{F}}(\mathbb{Z} G)$.

Let $\mathrm{E}$ be a model for $\mathrm{E} G$ and $\underline{\mathrm{E}}$ be a model for the universal space for proper $G$-actions. Then, $G \backslash F(\mathrm{E})$ is homeomorphic to $\mathfrak{N}(G,\{1\})$, and $\mathfrak{N}(G, \mathcal{F})$ is homeomorphic to $G \backslash F(\underline{E})$ by Theorem 3.7. The universal property of $\underline{E}$ implies that there is a $G-$ equivariant map, $f: \mathrm{E} \rightarrow \mathrm{E}$, that is unique up to $G$-homotopy equivalence. Then $F(f): F(\mathrm{E}) \rightarrow F(\underline{\mathrm{E}})$ induces a map $\bar{f}: G \backslash F(\mathrm{E}) \rightarrow G \backslash F(\underline{\mathrm{E}})$. Note that for a different choice of $f$, the induced map will be homotopy equivalent to $\bar{f}$. The corresponding map on homology groups is denoted $\bar{f}_{*}: H H_{*}(\mathbb{Z} G) \rightarrow H H_{*}^{\mathcal{F}}(\mathbb{Z} G)$. Recall that

$$
\overline{A_{\mathrm{E}}}: G \backslash(G \times \mathrm{E}) \rightarrow G \backslash F(\mathrm{E})
$$

is a homeomorphism, since $G$ acts freely and properly on E (Proposition 3.10). By Proposition 3.11, there is a homeomorphism

$$
h: \coprod_{C(g) \in \operatorname{conj}(G)} Z(g) \backslash \mathrm{E} \rightarrow G \backslash(G \times \mathrm{E}),
$$

which sends the orbit $Z(g) \cdot x$ to the orbit $G \cdot(g, x)$. This produces a map

$$
\phi: \coprod_{C(g) \in \operatorname{conj}(G)} Z(g) \backslash \mathrm{E} \rightarrow G \backslash F(\underline{\mathrm{E}}),
$$

where $\phi=\bar{f} \circ \bar{A}_{\mathrm{E}} \circ h$. That is, the image of $Z(g) \cdot x$ under $\phi$ is $G \cdot(f(x), g \cdot f(x))$, where $g$ is in $G$ and $x$ is in E. Thus, we have the following commutative diagram.

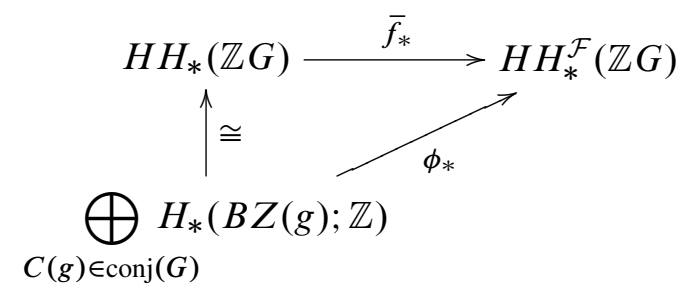

If $H$ is a finite group, then the Sullivan Conjecture, proved by Miller [17], implies that a map from $B H$ to a finite dimensional $\mathrm{CW}$ complex is null homotopic. If $\underline{\mathrm{E}}$ is finite 
dimensional, then $F(\underline{\mathrm{E}})$ is homotopy equivalent to a finite dimensional $\mathrm{CW}$ complex. Thus, if $Z(g)$ is finite, then the image of $H_{*}\left((B Z(g) ; \mathbb{Z})\right.$ under $\phi_{*}$ is zero.

For an illustrative example, consider the infinite dihedral group, $D_{\infty}=\langle a, b| a^{2}=$ $\left.1, a b a^{-1}=b^{-1}\right\rangle$. Let $\underline{E}=\mathbb{R}$, where $a$ acts by reflection through zero and $b$ acts by translation by 1 . That is, $a x=-x$ and $b x=x+1$. The space $F(\mathbb{R})$ is the image of $A_{\mathbb{R}}: D_{\infty} \times \mathbb{R} \rightarrow \mathbb{R} \times \mathbb{R}$. Thus, $F(\mathbb{R})=\left\{(x, g x) \mid x \in \mathbb{R}\right.$ and $\left.g \in D_{\infty}\right\}$. Every element of $D_{\infty}$ can be expressed as $b^{j}$ or $a b^{j}$, for some $j$ in $\mathbb{Z}$. Since $b^{j} x=x+j$ and $a b^{j} x=-x-j, F(\mathbb{R}) \subset \mathbb{R}^{2}$ is the union of the lines of slope 1 and -1 that cross the $y$-axis at an integer. A picture of $D_{\infty} \backslash F(\mathbb{R})$ is given in Figure 1 below.

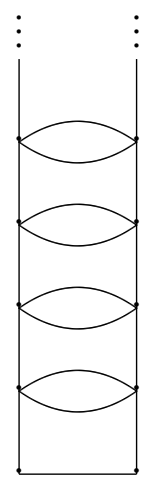

Figure 1: The space $D_{\infty} \backslash F(\mathbb{R})$

To see that this is in fact the picture, consider the diagonal action of $\langle b\rangle$ on $\mathbb{R}^{2}$. The orbit of the set

$$
D=\{(x, y) \mid x \in \mathbb{R} \text { and }-x-1 \leq y \leq-x+1\}
$$

under this action is all of $\mathbb{R}^{2}$. Observe that the lines $y=-x-1$ and $y=-x+1$ get identified in the quotient of $\mathbb{R}^{2}$ by $\langle b\rangle$ and that the rest of the set is mapped injectively into the quotient. Thus, $\langle b\rangle \backslash \mathbb{R}^{2}$ is an infinite cylinder. Since $a$ acts on the set $D$ by a rotation of $180^{\circ}$, we see that the quotient $D_{\infty} \backslash \mathbb{R}^{2}=\langle a\rangle \backslash\left(\langle b\rangle \backslash \mathbb{R}^{2}\right)$ is obtained from $\{(x, y) \in D \mid y \geq x\}$ by identifying the endpoints of the line segments $y=x+t$, where $t \geq 0$ (that is, the points $((-t-1) / 2,(t-1) / 2)$ and $((-t+1) / 2,(t+1) / 2))$, as well as by identifying the points $(x, x)$ and $(-x,-x)$, where $-1 / 2 \leq x \leq 1 / 2$. Thus, $D_{\infty} \backslash \mathbb{R}^{2}$ looks like an "infinite chisel," and $D_{\infty} \backslash F(\mathbb{R}) \subset D_{\infty} \backslash \mathbb{R}^{2}$ is as shown above.

The nontrivial finite subgroups of $D_{\infty}$ are of the form $\left\langle a b^{i}\right\rangle$, where $i \in \mathbb{Z}$. For each $i,\left\langle a b^{i}\right\rangle$ fixes $-i / 2 \in \mathbb{R}$. Beginning with the action of $D_{\infty}$ on $\mathbb{R}$, construct a model 
for E $D_{\infty}$ by replacing each half-integer with an $S^{\infty}$. Denote this "string of pearls" model for $\mathrm{E} D_{\infty}$ by $\mathrm{E}$, and let $f: \mathrm{E} \rightarrow \underline{\mathrm{E}}$ be the equivariant map that collapses each $S^{\infty}$ to a point. The conjugacy classes of $D_{\infty}$ are:

$$
\begin{aligned}
& C(1)=\{1\} \\
& C(a)=\left\{a b^{2 i}: i \in \mathbb{Z}\right\} \\
& C(a b)=\left\{a b^{2 i+1}: i \in \mathbb{Z}\right\} \\
& C\left(b^{j}\right)=\left\{b^{j}, b^{-j}\right\}, j \in \mathbb{N}
\end{aligned}
$$

The corresponding centralizers are:

$$
\begin{aligned}
& Z(1)=D_{\infty} \\
& Z(a)=\{1, a\} \\
& Z(a b)=\{1, a b\} \\
& Z\left(b^{j}\right)=\langle b\rangle, j \in \mathbb{N}
\end{aligned}
$$

Note that $D_{\infty} \backslash \mathrm{E}$ is an "interval" with an $\mathbb{R} P^{\infty}$ at each end; $\langle a\rangle \backslash \mathrm{E}$ is a "ray" that begins with an $\mathbb{R} P^{\infty}$ at 0 and has an $S^{\infty}$ at every positive half-integer; $\langle a b\rangle \backslash \mathrm{E}$ is a "ray" that begins with an $\mathbb{R} P^{\infty}$ at $1 / 2$ and has an $S^{\infty}$ at every other positive half-integer; and $\mathbb{Z} \backslash$ E is a "circle" with two $S^{\infty}$ 's in place of vertices.

The image of $\phi$ is broken into the pieces

$$
\begin{aligned}
\phi\left(D_{\infty} \cdot x\right) & =D_{\infty} \cdot(f(x), f(x)) \\
\phi(Z(a) \cdot x) & =D_{\infty} \cdot(f(x),-f(x)) \\
\phi(Z(a b) \cdot x) & =D_{\infty} \cdot(f(x),-f(x)-1) \\
\phi\left(Z\left(b^{j}\right) \cdot x\right) & =D_{\infty} \cdot(f(x), f(x)+j)
\end{aligned}
$$

where $j$ is a positive integer and $x \in \mathrm{E}$. Referring to Figure 1, the base of $D_{\infty} \backslash F(\underline{\mathrm{E}})$ is (4), the pieces (5) and (6) are the sides of $D_{\infty} \backslash F(\underline{E})$, and (7) provides each of the circles. Therefore, $\phi$ is a gluing of the disjoint pieces, $Z(g) \backslash$ E, after each $S^{\infty}$ and each $\mathbb{R} P^{\infty}$ is collapsed to a point. Observe that,

$$
\begin{aligned}
H H_{*}\left(\mathbb{Z} D_{\infty}\right) \cong H_{*}\left(\mathrm{~B} D_{\infty} ; \mathbb{Z}\right) & \oplus H_{*}(\mathrm{~B} Z(a) ; \mathbb{Z}) \\
& \oplus H_{*}(\mathrm{~B} Z(a b) ; \mathbb{Z}) \oplus \bigoplus_{j>0} H_{*}\left(\mathrm{~B} Z\left(b^{j}\right) ; \mathbb{Z}\right) .
\end{aligned}
$$

Since $Z(a) \cong \mathbb{Z} / 2 \cong Z(a b)$, the Sullivan Conjecture implies that the image of $H_{*}(\mathrm{~B} Z(a) ; \mathbb{Z})$ and $H_{*}(\mathrm{~B} Z(a b) ; \mathbb{Z})$ under $\phi_{*}$ is zero. By the above analysis, we 
have $\phi\left(\mathrm{B} D_{\infty}\right)=D_{\infty} \backslash \mathbb{R} \cong[0,1]$. Therefore, the image of $H_{i}\left(\mathrm{~B} D_{\infty} ; \mathbb{Z}\right)$ under $\phi_{i}$ is 0 , for $i \geq 1$. The rest of $H H_{i}\left(\mathbb{Z} D_{\infty}\right)$ is mapped injectively into $H H_{i}^{\mathcal{F}}\left(\mathbb{Z} D_{\infty}\right), i \geq 1$.

Classical Hochschild homology has been used to study the $K$-theory of group rings via the Dennis trace, dtr: $K_{*}(R G) \rightarrow H H_{*}(R G)$. In [15], Lück and Reich were able to determine how much of $K_{*}(\mathbb{Z} G)$ is detected by the Dennis trace. A natural question is to determine the composition of the Dennis trace with the map $\overline{f_{*}}: H H_{*}(\mathbb{Z} G) \rightarrow$ $H H_{*}^{\mathcal{F}}(\mathbb{Z} G)$. From Lück and Reich [15, p 595], we have the following commutative diagram

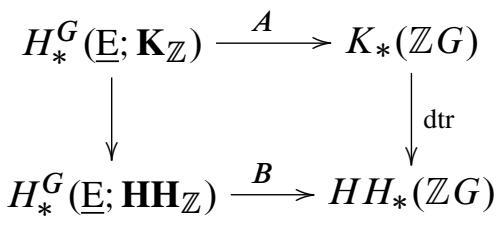

where the maps $A$ and $B$ are assembly maps in the equivariant homology theories with coefficients in the connective algebraic $K$-theory spectrum, $\mathbf{K}_{\mathbb{Z}}$, associated to $\mathbb{Z}$, and the Hochschild homology spectrum $\mathbf{H H}_{\mathbb{Z}}$, respectively. Each assembly map is induced by the collapse map $\underline{\mathrm{E}} \rightarrow \mathrm{pt}$. Lück and Reich used the composition of the Dennis trace with the assembly map in algebraic $K$-theory, dtr $\circ A$, to achieve their detection results. In particular, they observed [15, p 630] that the assembly map in Hochschild homology factors as:

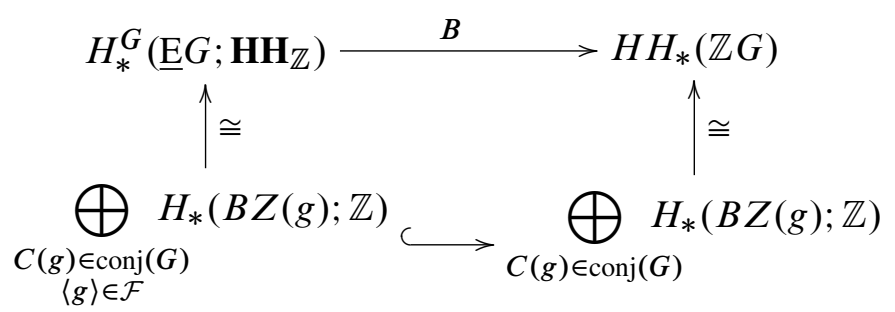

Given the discussion above, in the case $G=D_{\infty}$,

$$
H_{*}^{G}\left(\underline{\mathrm{E}} D_{\infty} ; \mathbf{H H}_{\mathbb{Z}}\right) \cong H_{*}\left(\mathrm{~B} D_{\infty} ; \mathbb{Z}\right) \oplus H_{*}(\mathrm{~B} Z(a) ; \mathbb{Z}) \oplus H_{*}(\mathrm{~B} Z(a b) ; \mathbb{Z}) .
$$

Therefore, $\overline{f_{*}} \circ B=0$, which implies that the image of $\overline{f_{*}} \circ \operatorname{dtr} \circ A$ is zero.

We conclude with speculation about a possible geometric application of the groups $H H_{*}^{\mathcal{F}}(\mathbb{Z} G)$. Associated to a parametrized family of self-maps of a manifold $M$, there are geometrically defined "intersection invariants," in particular, the framed bordism invariants of Hatcher and Quinn [10], which take values in abelian groups that are known to be related to the Hochschild homology groups $H H_{*}(\mathbb{Z} G)$, where $G$ is the fundamental group of $M$ [9]. It appears plausible that the groups $H H_{*}^{\mathcal{F}}(\mathbb{Z} G)$, where 
$\mathcal{F}$ is the family of finite subgroups, could play an analogous role in the yet to be developed homotopical intersection theory of orbifolds.

\section{References}

[1] R Ayala, F F Lasheras, A Quintero, The equivariant category of proper G-spaces, Rocky Mountain J. Math. 31 (2001) 1111-1132 MR1895288

[2] H Biller, Characterizations of proper actions, Math. Proc. Cambridge Philos. Soc. 136 (2004) 429-439 MR2040583

[3] C J R Borges, On stratifiable spaces, Pacific J. Math. 17 (1966) 1-16 MR0188982

[4] N Bourbaki, Elements of mathematics. General topology. Part 1, Hermann, Paris (1966) MR0205210

[5] G E Bredon, Introduction to compact transformation groups, Pure and Applied Math. 46, Academic Press, New York (1972) MR0413144

[6] R Cauty, Sur les espaces d'applications dans les CW-complexes, Arch. Math. (Basel) 27 (1976) 306-311 MR0407809

[7] J F Davis, W Lück, Spaces over a category and assembly maps in isomorphism conjectures in $K$ - and L-theory, $K$-Theory 15 (1998) 201-252 MR1659969

[8] J J Duistermaat, J A C Kolk, Lie groups, Universitext, Springer, Berlin (2000) MR1738431

[9] R Geoghegan, A Nicas, Parametrized Lefschetz-Nielsen fixed point theory and Hochschild homology traces, Amer. J. Math. 116 (1994) 397-446 MR1269610

[10] A Hatcher, F Quinn, Bordism invariants of intersections of submanifolds, Trans. Amer. Math. Soc. 200 (1974) 327-344 MR0353322

[11] B Hughes, Stratified path spaces and fibrations, Proc. Roy. Soc. Edinburgh Sect. A 129 (1999) 351-384 MR1686706

[12] J-L Loday, Cyclic homology, second edition, Grund. der Math. Wissenschaften [Fund. Principles of Math. Sciences] 301, Springer, Berlin (1998) MR1600246

[13] W Lück, Transformation groups and algebraic $K$-theory, Lecture Notes in Math. 1408, Springer, Berlin (1989) MR1027600Mathematica Gottingensis

[14] W Lück, Survey on classifying spaces for families of subgroups, from: "Infinite groups: geometric, combinatorial and dynamical aspects”, Progr. Math. 248, Birkhäuser, Basel (2005) 269-322 MR2195456

[15] W Lück, H Reich, Detecting K-theory by cyclic homology, Proc. London Math. Soc. (3) 93 (2006) 593-634 MR2266961

[16] A Lundell, S Weingram, The topology of $C W$ complexes, Van Nostrand Reinhold Co., New York (1969) 
[17] H Miller, The Sullivan conjecture on maps from classifying spaces, Ann. of Math. (2) 120 (1984) 39-87 MR750716

[18] J Milnor, Construction of universal bundles. I, Ann. of Math. (2) 63 (1956) 272-284 MR0077122

[19] R S Palais, The classification of $G$-spaces, Mem. Amer. Math. Soc. 36 (1960) iv+72 MR0177401

[20] R S Palais, On the existence of slices for actions of non-compact Lie groups, Ann. of Math. (2) 73 (1961) 295-323 MR0126506

[21] EH Spanier, Algebraic topology, McGraw-Hill Book Co., New York (1966) MR0210112

[22] R N Talbert, An isomorphism between Bredon and Quinn homology, Forum Math. 11 (1999) 591-616 MR1705904

Dept. of Mathematics \& Statistics, McMaster University Hamilton, ON L8S 4K1, Canada

Dept. of Mathematics \& Comp. Sci., St. John's University

8000 Utopia Pkwy, Jamaica, NY 11439, USA

nicas@mcmaster.ca, rosenthd@stjohns.edu

Received: 19 September 2007 Revised: 31 January 2008

Algebraic $\& \mathcal{G}$ Geometric Topology, Volume 8 (2008) 